Proceedings of the Edinburgh Mathematical Society (2007) 50, 293-324 (C)

DOI:10.1017/S0013091505000957 Printed in the United Kingdom

\title{
COMMUTATOR ESTIMATES AND R-FLOWS IN NON-COMMUTATIVE OPERATOR SPACES
}

\author{
BEN DE PAGTER ${ }^{1}$ AND FYODOR SUKOCHEV ${ }^{2}$ \\ ${ }^{1}$ Delft Institute of Applied Mathematics, Faculty of Electrical Engineering, \\ Mathematics and Computer Science, Delft University of Technology, \\ Mekelweg 4, 2628CD Delft, The Netherlands (b.depagter@ewi.tudelft.nl) \\ ${ }^{2}$ School of Informatics and Engineering, Flinders University of South Australia, \\ Bedford Park, 5042 South Australia, Australia (sukochev@infoeng.flinders.edu.au)
}

(Received 27 June 2005)

\begin{abstract}
The principal results in this paper are concerned with the description of domains of infinitesimal generators of strongly continuous groups of isometries in non-commutative operator spaces $E(\mathcal{M}, \tau)$, which are induced by $\mathbb{R}$-flows on $\mathcal{M}$. In particular, we are concerned with the description of operator functions which leave the domain of such generators invariant in all symmetric operator spaces, associated with a semi-finite von Neumann algebra $\mathcal{M}$ and a separable function space $E$ on $(0, \infty)$. Furthermore, we apply our results to the study of operator functions for which $[D, x] \in E(\mathcal{M}, \tau)$ implies that $[D, f(x)] \in E(\mathcal{M}, \tau)$, where $D$ is an unbounded self-adjoint operator. Our methods are partly based on the recently developed theory of double operator integrals in symmetric operator spaces and the theory of adjoint $C_{0}$-semigroups.
\end{abstract}

Keywords: non-commutative function spaces; commutators; double operator integrals

2000 Mathematics subject classification: Primary 46L52; 47B47

\section{Introduction}

The central theme of this paper is the investigation of the class of what could be called quantum differentiable functions. The principal aim is to determine which $C^{1}$-functions $f$ remain differentiable with respect to a (partially defined) symmetric derivation $\delta$ generated by an $\mathbb{R}$-flow on a semi-finite $W^{*}$-algebra (that is, $x=x^{*} \in \operatorname{Dom}(\delta)$ implies $f(x) \in \operatorname{Dom}(\delta))$. In the theory of Banach algebras and related parts of mathematical physics, especially quantum physics, the notion of a derivation of a Banach algebra $\mathcal{A}$ into an $\mathcal{A}$-bimodule $\mathcal{X}$ is important. The extensive development of this theory in the situation when $\mathcal{X}=\mathcal{A}$ and the algebra $\mathcal{A}$ is a $C^{*}$ - or $W^{*}$-algebra, and various applications to quantum physics, is presented in $[\mathbf{8}, \mathbf{3 2}]$. Since the time evolution of a quantum mechanical system in the $C^{*}$-algebraic formulation is modelled by a strongly continuous one-parameter group $\gamma=\left\{\gamma_{t}\right\}_{t \in \mathbb{R}}$ of $*$-automorphisms of the $C^{*}$-algebra $\mathcal{A}$, attention has been naturally focused on that part of the theory which studies unbounded derivations 
$\delta: \operatorname{Dom}(\delta) \rightarrow \mathcal{A}$, where $\delta$ is the closed densely defined infinitesimal generator of the group $\gamma$. The basic question in the study of derivations of this type is as follows.

For which scalar functions $f$ on $\mathbb{R}$ is $f(x) \in \operatorname{Dom}(\delta)$ whenever $x=x^{*} \in \operatorname{Dom}(\delta)$ ?

The answer to this question is important, because it allows the use of spectral theory to construct 'good' elements in the domain of the generator $\delta$. Moreover, this question is non-trivial and contains a number of subtle difficulties. For example, it was stated by Powers $[\mathbf{3 1}]$ that if $\delta$ is a closed symmetric derivation of a $C^{*}$-algebra $\mathcal{A}$ with dense domain $\operatorname{Dom}(\delta)$, then $f(x)$ belongs to $\operatorname{Dom}(\delta)$ for any $C^{1}$-function $f$ and $\|\delta(f(x))\| \leqslant\left\|f^{\prime}\right\|_{\infty}\|\delta(x)\|$, whenever $x=x^{*} \in \operatorname{Dom}(\delta)$. An explicit counterexample due to McIntosh [26] shows that this statement does not hold in general, although an amended version under the stronger assumption that $f \in C^{2}$ has been given in both [8] and $[\mathbf{3 2}]$. However, the proof given in [26, Theorem 4] contains a gap, recently noted and rectified in $[\mathbf{3 7}]$. Methods employed in [37] include the theory of so-called sun-dual semigroups and their generators, which goes back to the classical works of Phillips and will also be used in part here.

In this paper, we study the question above in the setting in which $\mathcal{X}$ is a bimodule of $\mathcal{A}$ that does not equal $\mathcal{A}$. Specifically, we consider here the situation in which $\mathcal{A}$ is represented as a semi-finite von Neumann algebra $\mathcal{M}$ (equipped with a semi-finite faithful normal trace $\tau$ ) on a Hilbert space $\mathcal{H}$, and $\mathcal{X}$ is a symmetric operator space $E(\mathcal{M}, \tau)$ associated with $\mathcal{M}$. This setting is in a certain sense classical, but relatively less studied than the case $\mathcal{X}=\mathcal{M}$.

Suppose that $(\mathcal{M}, \tau)$ is a semi-finite von Neumann algebra and let $\gamma=\left\{\gamma_{t}\right\}_{t \in \mathbb{R}}$ be an $\mathbb{R}$-flow in $\mathcal{M}$ (as defined at the beginning of $\S 4$ ). Let $E(\mathcal{M}, \tau)$ be the non-commutative symmetric space (consisting of, in general unbounded, $\tau$-measurable operators) associated with the separable Banach function space $E$ on $(0, \infty)$ and $\mathcal{M}$ (see $\S 2$ ). As is shown in Corollary 4.1, $\gamma$ induces a strongly continuous group $\gamma^{E}$ of isometries in the Banach space $E(\mathcal{M}, \tau)$. The infinitesimal generator of this group is denoted by $\delta^{E}$. The restriction of $\delta^{E}$ to $\operatorname{Dom}\left(\delta^{E}\right) \cap \mathcal{M}$ is indeed a derivation with values in $E(\mathcal{M}, \tau)$ (see Proposition 4.5). In the special case that $\mathcal{M}$ is the commutative von Neumann algebra $L_{\infty}(\mathbb{R})$ of all bounded Lebesgue measurable functions, equipped with integration as trace $\tau$ and acting via multiplication on the Hilbert space $\mathcal{H}=L_{2}(\mathbb{R})$, let the $\mathbb{R}$-flow $\gamma$ in $\mathcal{M}$ be given by forward translations. If, for example, $E=L_{p}(0, \infty)$ with $1 \leqslant p<\infty$, then $E(\mathcal{M}, \tau)=L_{p}(\mathbb{R})$ and the corresponding group $\gamma^{E}$ is the group of forward translations in $E$. Hence, in this case the domain of the infinitesimal generator $\delta^{E}$ is equal to the Sobolev space $W^{1, p}(\mathbb{R})$. Therefore, in the general setting, the domains $\operatorname{Dom}\left(\delta^{E}\right)$ could be considered as non-commutative extensions of Sobolev spaces.

In Theorem 5.7 a large class of functions $f: \mathbb{R} \rightarrow \mathbb{C}$ is exhibited with the property that $f(x) \in \operatorname{Dom}\left(\delta^{E}\right)$ whenever $x=x^{*} \in \operatorname{Dom}\left(\delta^{E}\right)$. Our main technical tool in the proof of this result is the theory of double operator integrals in the setting of symmetric operator spaces, recently developed in $[\mathbf{1 2}, \mathbf{1 3}]$, and strongly inspired by the corresponding theory in the setting of symmetrically normed ideals of compact operators, which was developed by Birman and Solomyak in [3-5]. In the latter setting, classes of operator-smooth 
functions have been recently studied by Kissin and Shulman [22-24]. We briefly recall a few notions from this theory in $\S 5$, below. In $\S \S 6$ and 7 we consider $\mathbb{R}$-flows $\gamma$ that are of the form $\gamma_{t}(x)=\mathrm{e}^{\mathrm{i} t D} x \mathrm{e}^{-\mathrm{i} t D}$, where $D$ is a self-adjoint operator in the underlying Hilbert space $\mathcal{H}$. As one would expect, the infinitesimal generator $\delta^{E}$ of the group $\gamma^{E}$ in $E(\mathcal{M}, \tau)$ has the form $\delta^{E}(x)=\mathrm{i}[D, x]$ (as usual, by $[\cdot, \cdot]$ we denote the commutator). However, since generally both operators $D$ and $x$ are unbounded, there is some difficulty in defining this commutator. If the operator $D$ is assumed to be $\tau$-measurable, then $[D, x]$ is well defined in the $*$-algebra $\tilde{\mathcal{M}}$ of all $\tau$-measurable operators. For this case a complete description of the domain of $\delta^{E}$ is given in Theorem 6.8. In the situation where $D$ is a general self-adjoint operator that induces an $\mathbb{R}$-flow in $\mathcal{M}$, we are able to obtain the equality $\delta^{E}(x)=\mathrm{i}[D, x]$ for a restricted class of $x \in \operatorname{Dom}\left(\delta^{E}\right)$ in Theorem 7.3. Finally, combining Theorems 6.8 and 7.3 with the result of Theorem 5.7, we obtain commutator estimates of the form $\|[D, f(x)]\|_{E(\mathcal{M}, \tau)} \leqslant C_{f}\|[D, x]\|_{E(\mathcal{M}, \tau)}$ (see Corollaries 6.9 and 7.5).

We start with some preliminary information concerning the theory of non-commutative integration in $\S 2$ and some additional results on convergence of measurable operators in $\S 3$, which will be needed in the remainder of the paper.

\section{Preliminaries on non-commutative integration}

In this section we introduce some notation and collect some results concerning the theory of non-commutative integration and symmetric operator spaces which will be used throughout the paper. Throughout this paper, we assume that $\mathcal{M}$ is a semi-finite von Neumann algebra on a Hilbert space $\mathcal{H}$, with a fixed faithful normal semi-finite trace $\tau$ and unit element $\mathbf{1}$.

Given a self-adjoint operator $a: \operatorname{Dom}(a) \rightarrow \mathcal{H}$ in the Hilbert space $\mathcal{H}$, the spectral measure of $a$ is denoted by $e^{a}$. We write $e_{\lambda}^{a}=e^{a}((-\infty, \lambda])$ for all $\lambda \in \mathbb{R}$. A linear operator $x: \operatorname{Dom}(x) \rightarrow \mathcal{H}$, with domain $\operatorname{Dom}(x) \subseteq \mathcal{H}$, is said to be affiliated with $\mathcal{M}$ if $u x=x u$ for all unitary operators $u$ in the commutant $\mathcal{M}^{\prime}$ of $\mathcal{M}$ (for the theory of von Neumann algebras see $[\mathbf{1 4}, \mathbf{2 0}, \mathbf{2 1}, \mathbf{3 3}-\mathbf{3 5}])$. A self-adjoint operator $a$ in $\mathcal{H}$ is affiliated with $\mathcal{M}$ if and only if $e^{a}(B) \in \mathcal{M}$ for all Borel sets $B \subseteq \mathbb{R}$ or, equivalently, $e_{\lambda}^{a} \in \mathcal{M}$ for all $\lambda \in \mathbb{R}$ (see, for example, [33, E.9.10, E.9.25]). If $x$ is a closed and densely defined linear operator in $\mathcal{H}$ with polar decomposition $x=v|x|$, then $x$ is affiliated with $\mathcal{M}$ if and only if $v \in \mathcal{M}$ and $|x|$ is affiliated with $\mathcal{M}$ (see, for example, [33, § 9.29]; [35, § IX.2].

A closed and densely defined linear operator $x$, affiliated with $\mathcal{M}$, is called $\tau$-measurable if there exists $\lambda>0$ such that $\tau\left(e^{|x|}(\lambda, \infty)\right)<\infty$. The set $\tilde{\mathcal{M}}$ of all $\tau$-measurable operators is a $*$-algebra with the sum and product defined as the closure of the algebraic sum and product, respectively. For $\varepsilon, \delta>0$ we denote by $N(\varepsilon, \delta)$ the set of all $x \in \tilde{\mathcal{M}}$ for which there exists an orthogonal projection $p \in \mathcal{M}$ such that $p(\mathcal{H}) \subseteq \operatorname{Dom}(x),\|x p\| \leqslant \varepsilon$ and $\tau(\mathbf{1}-p) \leqslant \delta$. The sets $\{N(\varepsilon, \delta): \varepsilon, \delta>0\}$ are a base at 0 for a metrizable Hausdorff vector space topology in $\tilde{\mathcal{M}}$, which is called the measure topology. Convergence with respect to this topology is referred to as convergence in measure. Equipped with the measure topology, $\tilde{\mathcal{M}}$ is a complete topological $*$-algebra in which $\mathcal{M}$ is dense. In fact, if $0 \leqslant x \in \tilde{\mathcal{M}}$, then $\left\{x e_{\lambda}^{x}\right\}_{\lambda \geqslant 0}$, which is contained in $\mathcal{M}$, converges in measure to $x$ as 
$\lambda \rightarrow \infty$. Furthermore, if $\left\{x_{n}\right\}_{n=1}^{\infty}$ is a sequence in $\tilde{\mathcal{M}}$, then

$$
x_{n} \rightarrow 0 \text { in measure } \Longleftrightarrow \tau\left(e^{\left|x_{n}\right|}(\lambda, \infty)\right) \rightarrow 0 \text { as } n \rightarrow \infty \text { for all } \lambda>0 .
$$

The proofs of these facts can be found in $[\mathbf{1 8}, \mathbf{2 7}, \mathbf{3 6}]$ (see also [35]).

Given $x \in \tilde{\mathcal{M}}$, the generalized singular-value function $\mu(x):[0, \infty) \rightarrow[0, \infty]$ of $x$ is defined by

$$
\mu_{t}(x)=\inf \left\{\lambda \geqslant 0: \tau\left(e^{|x|}(\lambda, \infty)\right) \leqslant t\right\}
$$

for all $t \geqslant 0$. Note that $\mu_{t}(x)<\infty$ for all $t>0$ and that $\mu_{0}(x)<\infty$ if and only if $x \in \mathcal{M}$, in which case $\mu_{0}(x)=\|x\|$. A detailed study of the properties of these generalized singular-value functions can be found in $[\mathbf{1 8}]$.

Furthermore, we define

$$
\tilde{\mathcal{M}}_{0}=\left\{x \in \tilde{\mathcal{M}}: \mu_{t}(x) \rightarrow 0 \text { as } t \rightarrow \infty\right\} .
$$

If $x \in \tilde{\mathcal{M}}$, then $x \in \tilde{\mathcal{M}}_{0}$ if and only if $\tau\left(e^{|x|}(\lambda, \infty)\right)<\infty$ for all $\lambda>0$.

For the general theory of rearrangement invariant Banach function spaces we refer the reader to $[\mathbf{2}, \mathbf{2 5}]$. We consider these spaces on the interval $(0, \infty)$ equipped with the Lebesgue measure $\mathrm{d} t$. The space of all (equivalence classes of) real-valued measurable functions on $(0, \infty)$ is denoted by $S(0, \infty)$. For $f \in S(0, \infty)$ we denote by $f^{*}$ the decreasing rearrangement of the function $|f|$. Recall that a Banach space $E$, equipped with a norm $\|\cdot\|_{E}$, is called a rearrangement invariant (r.i.) Banach function space $(B F S)$ on $(0, \infty)$ if $\{0\} \neq E \subseteq S(0, \infty)$ and, if $f \in E, g \in S(0, \infty), g^{*} \leqslant f^{*}$, then $g \in E$ and $\|g\|_{E} \leqslant\|f\|_{E}$. Special examples of such BFSs are the spaces $L_{p}(0, \infty), 1 \leqslant p \leqslant \infty$, equipped with their usual norm $\|\cdot\|_{p}$. All these spaces $E$ satisfy $L_{1} \cap L_{\infty}(0, \infty) \subseteq E \subseteq\left(L_{1}+L_{\infty}\right)(0, \infty)$, with continuous embeddings. We recall that the norm in $E$ is said to be order continuous if $f_{n} \downarrow 0$ in $E$ implies that $\left\|f_{n}\right\|_{E} \downarrow 0$. Order continuity of the norm is equivalent to separability of the Banach space $E$. An r.i. BFS $E$ is called symmetric if $f, g \in E$ and $g \prec \prec f$ imply that $\|g\|_{E} \leqslant\|f\|_{E}$ (it is called fully symmetric if it has the additional property that $f \in E, g \in S(0,1)$ and $g \prec \prec f$ imply that $g \in E)$. Here $g \prec \prec f$ means that the function $g$ is submajorized by $f$, that is,

$$
\int_{0}^{t} g^{*}(s) \mathrm{d} s \leqslant \int_{0}^{t} f^{*}(s) \mathrm{d} s
$$

for all $t \geqslant 0$. A separable r.i. BFS $E$ is necessarily fully symmetric [25, Theorems II 4.3 and II 4.10].

For any symmetric BFS $E$ on $(0, \infty)$ the corresponding non-commutative space $E(\mathcal{M}, \tau)$ associated with $(\mathcal{M}, \tau)$ is defined by

$$
E(\mathcal{M}, \tau)=\{x \in \tilde{\mathcal{M}}: \mu(x) \in E\},
$$

equipped with the norm given by $\|x\|_{E(\mathcal{M}, \tau)}=\|\mu(x)\|_{E}$ for all $x \in E(\mathcal{M}, \tau)$. It can be shown that $\left(E(\mathcal{M}, \tau),\|\cdot\|_{E(\mathcal{M}, \tau)}\right)$ is indeed a Banach space (see [15] for the details), which is called a non-commutative symmetric space (or a symmetric operator space). In 
particular, if $E=L_{p}(0, \infty), 1 \leqslant p \leqslant \infty$, then $E(\mathcal{M}, \tau)=L_{p}(\mathcal{M}, \tau)$ is the corresponding non-commutative $L_{p}$-space. The norm in $L_{p}(\mathcal{M}, \tau)$ is usually denoted by $\|\cdot\|_{p}$. Furthermore, we note that $L_{\infty}(\mathcal{M}, \tau)=\mathcal{M}$ and for this reason the norm in $\mathcal{M}$ is also denoted by $\|\cdot\|_{\infty}$. Observe that every non-commutative symmetric space $E(\mathcal{M}, \tau)$ satisfies

$$
L_{1} \cap L_{\infty}(\mathcal{M}, \tau) \subseteq E(\mathcal{M}, \tau) \subseteq\left(L_{1}+L_{\infty}\right)(\mathcal{M}, \tau),
$$

with continuous embeddings. The trace $\tau$ on $\mathcal{M}^{+}$extends uniquely to an additive, positively homogeneous, unitarily invariant and normal functional $\tilde{\tau}: \tilde{\mathcal{M}}^{+} \rightarrow[0, \infty]$, which is given by

$$
\tilde{\tau}(a)=\int_{0}^{\infty} \mu_{t}(a) \mathrm{d} t \quad \text { for all } a \in \tilde{\mathcal{M}}^{+}
$$

(for the details see, for example, $[\mathbf{1 6}, \S 3]$ ). For convenience, we denote this extension $\tilde{\tau}$ again by $\tau$. An operator $x \in \tilde{\mathcal{M}}$ belongs to $L_{1}(\mathcal{M}, \tau)$ if and only if $\tau(|x|)<\infty$. The restriction of $\tau$ to $L_{1}(\mathcal{M}, \tau)^{+}$induces a bounded linear functional on the space $L_{1}(\mathcal{M}, \tau)$, which will also be denoted by $\tau$. The norm on $L_{1}(\mathcal{M}, \tau)$ is given by $\|x\|_{1}=\tau(|x|)$ for all $x \in L_{1}(\mathcal{M}, \tau)$.

For any non-commutative symmetric space $E(\mathcal{M}, \tau)$, the Köthe dual space is defined by

$$
E(\mathcal{M}, \tau)^{\times}=\left\{y \in \tilde{\mathcal{M}}: x y \in L_{1}(\mathcal{M}, \tau) \text { for all } x \in E(\mathcal{M}, \tau)\right\},
$$

which is equipped with the norm given by

$$
\|y\|_{E(\mathcal{M}, \tau) \times}=\sup \left\{|\tau(x y)|: x \in E(\mathcal{M}, \tau),\|x\|_{E(\mathcal{M}, \tau)} \leqslant 1\right\}
$$

for all $y \in E(\mathcal{M}, \tau)^{\times}$. The Banach space $\left(E(\mathcal{M}, \tau)^{\times},\|\cdot\|_{E(\mathcal{M}, \tau)^{\times}}\right)$is a non-commutative symmetric space. Actually,

$$
E(\mathcal{M}, \tau)^{\times}=E^{\times}(\mathcal{M}, \tau)=\left\{y \in \tilde{\mathcal{M}}: \mu(y) \in E^{\times}\right\}
$$

and $\|y\|_{E(\mathcal{M}, \tau) \times}=\|\mu(y)\|_{E \times}$ for all $y \in E(\mathcal{M}, \tau)^{\times}[\mathbf{1 6}$, Theorem 5.6]. As in the commutative situation, $E(\mathcal{M}, \tau)^{\times}$may be identified with a closed subspace of the Banach space dual $E(\mathcal{M}, \tau)^{*}$ via the duality pairing given by

$$
\langle x, y\rangle=\tau(x y)=\tau(y x)
$$

for all $x \in E(\mathcal{M}, \tau)$ and $y \in E(\mathcal{M}, \tau)^{\times}$. For the details concerning the non-commutative duality we refer the reader to [16]. In particular, we recall that if $E$ has order-continuous norm, then $E(\mathcal{M}, \tau)$ has order-continuous norm as well (that is, $x_{\alpha} \downarrow 0$ in $E(\mathcal{M}, \tau)$ implies that $\left\|x_{\alpha}\right\|_{E} \downarrow 0$ ), which implies that $E(\mathcal{M}, \tau)^{*}=E(\mathcal{M}, \tau)^{\times}$(see [16, Proposition 3.6 and Theorem 5.11] and [10]). Furthermore, in this case, the subspace $L_{1} \cap L_{\infty}(\mathcal{M}, \tau)$ is dense in $E(\mathcal{M}, \tau)$ (see [16, Proposition 2.8]) and $E(\mathcal{M}, \tau) \subseteq \tilde{\mathcal{M}}_{0}$. 


\section{Some additional results concerning measurable operators}

The real subspace of $\mathcal{M}$ consisting of all self-adjoint elements is denoted by $\mathcal{M}_{h}$. Similarly, $\tilde{\mathcal{M}}_{h}$ denotes the real subspace of all self-adjoint elements in $\tilde{\mathcal{M}}$. If $a \in \tilde{\mathcal{M}}_{h}$ and $f: \mathbb{R} \rightarrow \mathbb{C}$ is a Borel function, then the normal operator $f(a)$ is defined via the functional calculus by

$$
f(a)=\int_{\mathbb{R}} f(\lambda) \mathrm{d} e^{a}(\lambda) .
$$

Recall that the spectral measure $e^{f(a)}$ of $f(a)$ is given by

$$
e^{f(a)}(B)=e^{a}\left(f^{-1}(B)\right), \quad B \in \operatorname{Bor}(\mathbb{C}),
$$

where Bor $(\mathbb{C})$ denotes the $\sigma$-algebra of all Borel subsets of $\mathbb{C}$. Since $e^{a}$ takes its values in the projections of $\mathcal{M}$, it is clear from $(3.1)$ that $e^{f(a)}(B) \in \mathcal{M}$ for all $B \in \operatorname{Bor}(\mathbb{C})$, and so $f(a)$ is affiliated with $\mathcal{M}$. The next lemma gives sufficient conditions on the function $f$ to guarantee that $f(a)$ is $\tau$-measurable.

Lemma 3.1. If $a \in \tilde{\mathcal{M}}_{h}$ and if $f: \mathbb{R} \rightarrow \mathbb{C}$ is a Borel function which is bounded on bounded subsets of $\mathbb{R}$, then $f(a) \in \tilde{\mathcal{M}}$.

Proof. We must show that $\tau\left(e^{|f(a)|}(s, \infty)\right)<\infty$ for some $s>0$. Note that it follows from (3.1) that

$$
e^{|f(a)|}(s, \infty)=e^{a}(\{\lambda \in \mathbb{R}:|f(\lambda)|>s\})
$$

for all $s>0$. Since $a \in \tilde{\mathcal{M}}_{h}$, there exists $t>0$ such that $\tau\left(e^{|a|}(t, \infty)\right)<\infty$ and, since $f$ is bounded on the interval $[-t, t]$, there exists a constant $C>0$ such that $|f(\lambda)| \leqslant C$ for all $\lambda \in[-t, t]$. This implies that

$$
\{\lambda \in \mathbb{R}:|f(\lambda)|>C\} \subseteq\{\lambda \in \mathbb{R}:|\lambda|>t\}
$$

and so it follows from (3.2) that

$$
\begin{aligned}
e^{|f(a)|}(C, \infty) & =e^{a}(\{\lambda \in \mathbb{R}:|f(\lambda)|>C\}) \\
& \leqslant e^{a}(\{\lambda \in \mathbb{R}:|\lambda|>t\}) \\
& =e^{|a|}(t, \infty) .
\end{aligned}
$$

Hence, $\tau\left(e^{|f(a)|}(C, \infty)\right) \leqslant \tau\left(e^{|a|}(t, \infty)\right)<\infty$.

Proposition 3.2. Suppose that $a \in \tilde{\mathcal{M}}_{h}$ and that $f, f_{n}: \mathbb{R} \rightarrow \mathbb{C}(n=1,2, \ldots)$ are Borel functions that are bounded on bounded subsets of $\mathbb{R}$. Assume that $f_{n} \rightarrow f$ as $n \rightarrow \infty$ uniformly on bounded subsets of $\mathbb{R}$. Then $f_{n}(a) \rightarrow f(a)$ in measure as $n \rightarrow \infty$.

Proof. Replacing $f_{n}$ by $f_{n}-f$, it is sufficient to show that $f_{n}(a) \rightarrow 0$ as $n \rightarrow \infty$ in measure whenever $f_{n} \rightarrow 0$ uniformly on bounded subsets of $\mathbb{R}$. As observed in (2.1), we have to show that $\tau\left(e^{\left|f_{n}(a)\right|}(\lambda, \infty)\right) \rightarrow 0$ as $n \rightarrow \infty$ for all $\lambda>0$. Fix $\lambda>0$ and let $\varepsilon>0$ be given. Since $a$ is $\tau$-measurable, we know that

$$
\lim _{t \rightarrow \infty} \tau\left(e^{|a|}(t, \infty)\right)=0 .
$$


Hence, there exists $R>0$ such that $\tau\left(e^{|a|}(R, \infty)\right)<\varepsilon$. Note that

$$
e^{|a|}(R, \infty)=e^{a}(\{\mu \in \mathbb{R}:|\mu|>R\}) .
$$

Since $f_{n} \rightarrow 0$ uniformly on $[-R, R]$, there exists $N \in \mathbb{N}$ such that $\left|f_{n}(\mu)\right|<\lambda$ for all $\mu \in[-R, R]$ and all $n \geqslant N$. This implies that

$$
\left\{\mu \in \mathbb{R}:\left|f_{n}(\mu)\right|>\lambda\right\} \subseteq\{\mu \in \mathbb{R}:|\mu|>R\}
$$

for all $n \geqslant N$. Hence, for all $n \geqslant N$, we find that

$$
\begin{aligned}
e^{\left|f_{n}(a)\right|}(\lambda, \infty) & =e^{a}\left(\left\{\mu \in \mathbb{R}:\left|f_{n}(\mu)\right|>\lambda\right\}\right) \\
& \leqslant e^{a}(\{\mu \in \mathbb{R}:|\mu|>R\}) \\
& =e^{|a|}(R, \infty)
\end{aligned}
$$

This implies that

$$
\tau\left(e^{\left|f_{n}(a)\right|}(\lambda, \infty)\right) \leqslant \tau\left(e^{|a|}(R, \infty)\right)<\varepsilon
$$

for all $n \geqslant N$.

Note the following consequence of the above proposition. Suppose that $a \in \tilde{\mathcal{M}}_{h}$ and that $f: \mathbb{R} \rightarrow \mathbb{C}$ is a Borel function that is bounded on bounded subsets of $\mathbb{R}$. For $n \in \mathbb{N}$ we define $f_{n}=f \chi_{\{|f| \leqslant n\}}$. Evidently, $f_{n} \rightarrow f$ uniformly on bounded subsets of $\mathbb{R}$ and, consequently, $f_{n}(a) \rightarrow f(a)$ in measure as $n \rightarrow \infty$. Note that each $f_{n}$ is bounded and so $f_{n}(a) \in \mathcal{M}$ for all $n$.

Suppose that $\alpha: \mathcal{M} \rightarrow \mathcal{M}$ is a $*$-automorphism. If $a$ is a self-adjoint operator on $\mathcal{H}$, affiliated with $\mathcal{M}$, then the measure $\alpha\left(e^{a}\right)$, defined by $\alpha\left(e^{a}\right)(B)=\alpha\left(e^{a}(B)\right)$ for all $B \in$ $\operatorname{Bor}(\mathbb{R})$, is a spectral measure taking its values in $\mathcal{M}$. If $f: \mathbb{R} \rightarrow \mathbb{C}$ is a bounded Borel function, then $f(a) \in \mathcal{M}$. We claim that

$$
\alpha(f(a))=\int_{\mathbb{R}} f(\lambda) \mathrm{d} \alpha\left(e^{a}\right) .
$$

Indeed, equality (3.3) is easily verified if $f$ is a simple function. The general case now follows via uniform approximation of $f$ by simple functions. In particular, if $a \in \mathcal{M}_{h}$, then the spectral measure $e^{a}$ is supported on any closed interval $[-R, R]$ containing the spectrum of $a$. Applying (3.3) to the function $f$ given by $f(\lambda)=\lambda \chi_{[-R, R]}(\lambda)$, we find that

$$
\alpha(a)=\int_{\mathbb{R}} \lambda \mathrm{d} \alpha\left(e^{a}\right) .
$$

By the uniqueness of the spectral measure, this implies that $e^{\alpha(a)}=\alpha\left(e^{a}\right)$. Consequently,

$$
f(\alpha(a))=\int_{\mathbb{R}} f(\lambda) \mathrm{d} \alpha\left(e^{a}\right)=\alpha(f(a))
$$

for all bounded Borel functions $f: \mathbb{R} \rightarrow \mathbb{C}$.

Now we assume that $\alpha: \mathcal{M} \rightarrow \mathcal{M}$ is a $*$-automorphism which is in addition trace preserving, that is, $\tau(\alpha(a))=\tau(a)$ for all $0 \leqslant a \in \mathcal{M}$. The next proposition shows that such an automorphism extends to a $*$-automorphism of $\tilde{\mathcal{M}}$. 
Proposition 3.3. If $\alpha$ is a $\tau$-preserving *-automorphism of $\mathcal{M}$, then $\alpha$ extends uniquely to a $*$-automorphism $\tilde{\alpha}: \tilde{\mathcal{M}} \rightarrow \tilde{\mathcal{M}}$, which is continuous with respect to the measure topology. Moreover,

(i) if $f: \mathbb{R} \rightarrow \mathbb{C}$ is a Borel function, which is bounded on bounded subsets of $\mathbb{R}$, and if $a \in \tilde{\mathcal{M}}_{h}$, then $\tilde{\alpha}(f(a))=f(\tilde{\alpha}(a))$;

(ii) $\tilde{\alpha}$ is rearrangement preserving, that is, $\mu(\tilde{\alpha}(x))=\mu(x)$ for all $x \in \tilde{\mathcal{M}}$;

(iii) $\tilde{\alpha}$ is trace preserving, that is, $\tau(\tilde{\alpha}(a))=\tau(a)$ for all $0 \leqslant a \in \tilde{\mathcal{M}}$;

(iv) if $z \in L_{1}(\mathcal{M}, \tau)$, then $\tilde{\alpha}(z) \in L_{1}(\mathcal{M}, \tau)$ and $\tau(\tilde{\alpha}(z))=\tau(z)$.

Proof. As observed above (see (3.4)), for any $0 \leqslant a \in \mathcal{M}$ the spectral measure of $\alpha(a)$ is given by $e^{\alpha(a)}=\alpha\left(e^{a}\right)$ and consequently, it follows from the definition of the generalized singular-value function that $\mu_{t}(\alpha(a))=\mu_{t}(a)$ for all $t \geqslant 0$. Since $|\alpha(x)|=\alpha(|x|)$ for all $x \in \mathcal{M}$, we conclude that $\mu(\alpha(x))=\mu(x)$ for all $x \in \mathcal{M}$. A sequence $\left\{x_{n}\right\}_{n=1}^{\infty}$ in $\mathcal{M}$ converges to 0 in measure if and only if $\mu_{t}\left(x_{n}\right) \rightarrow 0$ as $n \rightarrow \infty$ for all $t>0$ and so we infer that $\alpha$ is continuous with respect to this topology. Since $\tilde{\mathcal{M}}$ is complete and $\mathcal{M}$ is dense in $\tilde{\mathcal{M}}$ with respect to the measure topology, $\alpha$ has a unique continuous extension $\tilde{\alpha}: \tilde{\mathcal{M}} \rightarrow \tilde{\mathcal{M}}$, which is a $*$-automorphism.

For the proof of (i), define the self-adjoint operator $b$ in $\mathcal{H}$ by

$$
b=\int_{\mathbb{R}} \lambda \mathrm{d} \alpha\left(e^{a}\right)(\lambda) .
$$

Since the spectral measure $\alpha\left(e^{a}\right)$ takes its values in $\mathcal{M}$, it is clear that $b$ is affiliated with $\mathcal{M}$ and $e^{b}=\alpha\left(e^{a}\right)$. Actually, $b \in \tilde{\mathcal{M}}_{h}$. Indeed, since $a$ is $\tau$-measurable, there exists $s>0$ such that $\tau\left(e^{|a|}(s, \infty)\right)<\infty$. Furthermore,

$$
\begin{aligned}
e^{|b|}(s, \infty) & =e^{b}(\{\lambda \in \mathbb{R}:|\lambda|>s\}) \\
& =\alpha\left(e^{a}(\{\lambda \in \mathbb{R}:|\lambda|>s\})\right) \\
& =\alpha\left(e^{|a|}(s, \infty)\right)
\end{aligned}
$$

and so $\tau\left(e^{|b|}(s, \infty)\right)=\tau\left(\alpha\left(e^{|a|}(s, \infty)\right)\right)=\tau\left(e^{|a|}(s, \infty)\right)<\infty$. Hence, $b$ is $\tau$-measurable. Now assume that $f: \mathbb{R} \rightarrow \mathbb{C}$ is a Borel function, which is bounded on bounded subsets of $\mathbb{R}$. Let $\left\{f_{n}\right\}_{n=1}^{\infty}$ be a sequence of bounded Borel functions such that $f_{n} \rightarrow f$ uniformly on compact subsets of $\mathbb{R}$ as $n \rightarrow \infty$. It follows from (3.3) that $\tilde{\alpha}\left(f_{n}(a)\right)=\alpha\left(f_{n}(a)\right)=f_{n}(b)$ for all $n$. Moreover, by Proposition 3.2, $f_{n}(a) \rightarrow f(a)$ and $f_{n}(b) \rightarrow f(b)$ in measure as $n \rightarrow \infty$. From the continuity of $\tilde{\alpha}$ with respect to the measure topology, it follows that $\tilde{\alpha}\left(f_{n}(a)\right) \rightarrow \tilde{\alpha}(f(a))$ as $n \rightarrow \infty$. Therefore, we may conclude that $\tilde{\alpha}(f(a))=f(b)$. Applying this equality in particular to the function $f(\lambda)=\lambda$, we see that $b=\tilde{\alpha}(a)$, and hence, $\tilde{\alpha}(f(a))=f(\tilde{\alpha}(a))$. This proves (i).

It follows in particular from (i) that $e^{\tilde{\alpha}(a)}=\alpha\left(e^{a}\right)$ for all $a \in \tilde{\mathcal{M}}_{h}$. Since $\alpha$ is trace preserving, this implies that $\mu(\tilde{\alpha}(a))=\mu(a)$ for all $0 \leqslant a \in \tilde{\mathcal{M}}$. Consequently,

$$
\mu(\tilde{\alpha}(x))=\mu(|\tilde{\alpha}(x)|)=\mu(\tilde{\alpha}(|x|))=\mu(|x|)=\mu(x)
$$


for all $x \in \tilde{\mathcal{M}}$, and this shows that (ii) holds. Since

$$
\tau(a)=\int_{0}^{\infty} \mu_{t}(a) \mathrm{d} t \quad \text { for all } 0 \leqslant a \in \tilde{\mathcal{M}},
$$

(iii) is an immediate consequence of (ii). Finally, since every $z \in L_{1}(\mathcal{M}, \tau)$ is a linear combination of four positive elements of $L_{1}(\mathcal{M}, \tau)$, statement (iv) is now evident.

\section{4. $\mathbb{R}$-flows on symmetric operator spaces}

An $\mathbb{R}$-flow on $(\mathcal{M}, \tau)$ is an ultra-weakly continuous (equivalently, $\sigma\left(\mathcal{M}, L_{1}\right)$-continuous) representation $\gamma=\left\{\gamma_{t}\right\}_{t \in \mathbb{R}}$ of $\mathbb{R}$ on $\mathcal{M}$ by trace preserving $*$-automorphisms of $\mathcal{M}$. It follows from Proposition 3.3 that $\gamma$ has a canonical extension to a group $\tilde{\gamma}=\left\{\tilde{\gamma}_{t}\right\}_{t \in \mathbb{R}}$ of *-automorphisms on $\tilde{\mathcal{M}}$. Furthermore, each $\tilde{\gamma}_{t}$ is rearrangement preserving, which allows us to define, for any symmetric BFS $E$ on $(0, \infty)$, the group $\gamma^{E}=\left\{\gamma_{t}^{E}\right\}_{t \in \mathbb{R}}$ of isometries in the corresponding non-commutative space $E(\mathcal{M}, \tau)$, where

$$
\gamma_{t}^{E}=\left.\left(\tilde{\gamma}_{t}\right)\right|_{E(\mathcal{M}, \tau)}
$$

for all $t \in \mathbb{R}$. Similarly, the group $\gamma^{E^{\times}}$is defined on the Köthe dual $E(\mathcal{M}, \tau)^{\times}$of $E(\mathcal{M}, \tau)$. Since $\tilde{\gamma}$ is trace preserving, it follows that

$$
\begin{aligned}
\tau\left(\gamma_{t}^{E}(x) y\right) & =\tau\left(\tilde{\gamma}_{t}(x) y\right)=\tau\left(\tilde{\gamma}_{-t}\left(\tilde{\gamma}_{t}(x) y\right)\right) \\
& =\tau\left(x\left(\tilde{\gamma}_{-t} y\right)\right)=\tau\left(x\left(\gamma_{-t}^{E^{\times}} y\right)\right)
\end{aligned}
$$

for all $x \in E(\mathcal{M}, \tau)$ and $y \in E(\mathcal{M}, \tau)^{\times}$. In other words,

$$
\left.\left(\left(\gamma_{t}^{E}\right)^{*}\right)\right|_{E(\mathcal{M}, \tau) \times}=\gamma_{-t}^{E^{\times}}
$$

for all $t \in \mathbb{R}$, where we consider $E(\mathcal{M}, \tau)^{\times} \subseteq E(\mathcal{M}, \tau)^{*}$ via trace duality (2.3). If $E=$ $L_{p}(0, \infty)$, with $1 \leqslant p \leqslant \infty$, then we denote the group $\gamma^{L_{p}}$ on $L_{p}(\mathcal{M}, \tau)$ simply by $\gamma^{p}$. In general, the group $\gamma^{E}$ is not strongly continuous in the Banach space $E(\mathcal{M}, \tau)$. However, the next proposition shows that separability of the BFS $E$ implies strong continuity.

Proposition 4.1. If $\gamma$ is an $\mathbb{R}$-flow $(\mathcal{M}, \tau)$ and $E$ is a separable $B F S$ on $(0, \infty)$, then $\gamma^{E}$ is a strongly continuous group on the symmetric operator space $E(\mathcal{M}, \tau)$.

Proof. By [11, Proposition 1.23], it suffices to prove that $\gamma^{E}$ is $\sigma\left(E(\mathcal{M}, \tau), E(\mathcal{M}, \tau)^{*}\right)$ continuous. Since $E$ has order-continuous norm, $E(\mathcal{M}, \tau)^{*}=E(\mathcal{M}, \tau)^{\times}$, as observed at the end of $\S 2$. Hence, it is sufficient to show that the function $t \mapsto \tau\left(\gamma_{t}^{E}(x) y\right)$ is continuous for all $x \in E(\mathcal{M}, \tau)$ and $y \in E(\mathcal{M}, \tau)^{\times}$.

First we consider the special situation that $x \in L_{1}(\mathcal{M}, \tau) \cap \mathcal{M}$. Take $y \in E(\mathcal{M}, \tau)^{\times}$ and write $y=y_{1}+y_{\infty}$ with $y_{1} \in L_{1}(\mathcal{M}, \tau)$ and $y_{\infty} \in \mathcal{M}$. For $t \in \mathbb{R}$ we have

$$
\begin{aligned}
\tau\left(\gamma_{t}^{E}(x) y\right) & =\tau\left(\gamma_{t}^{\infty}(x) y_{1}\right)+\tau\left(\gamma_{t}^{1}(x) y_{\infty}\right) \\
& =\tau\left(\gamma_{t}^{\infty}(x) y_{1}\right)+\tau\left(x \gamma_{-t}^{\infty}\left(y_{\infty}\right)\right) .
\end{aligned}
$$


By hypothesis, the functions $t \mapsto \tau\left(\gamma_{t}^{\infty}(x) y_{1}\right)$ and $t \mapsto \tau\left(x \gamma_{-t}^{\infty}\left(y_{\infty}\right)\right)$ are continuous, and so the function $t \mapsto \tau\left(\gamma_{t}^{L_{1} \cap L_{\infty}}(x) y\right)$ is continuous.

Next we consider the general case. Fix $y \in E(\mathcal{M}, \tau)^{\times}$. For $x \in E(\mathcal{M}, \tau)$, define the function $f_{x}: \mathbb{R} \rightarrow \mathbb{C}$ by $f_{x}(t)=\tau\left(\gamma_{t}^{E}(x) y\right)$ for all $t \in \mathbb{R}$. We have to show that $f_{x}$ is continuous. Fix $x \in E(\mathcal{M}, \tau)$. Since $E(\mathcal{M}, \tau)$ has order-continuous norm, $L_{1}(\mathcal{M}, \tau) \cap \mathcal{M}$ is dense in $E(\mathcal{M}, \tau)$ and so, for each $n \in \mathbb{N}$, there exists $z_{n} \in L_{1}(\mathcal{M}, \tau) \cap \mathcal{M}$ such that $\left\|x-z_{n}\right\|_{E(\mathcal{M}, \tau)} \leqslant 1 / n$. For all $t \in \mathbb{R}$ we have

$$
\begin{aligned}
\left|f_{x}(t)-f_{z_{n}}(t)\right| & =\left|\tau\left(\gamma_{t}^{E}(x) y\right)-\tau\left(\gamma_{t}^{E}\left(z_{n}\right) y\right)\right|=\left|\tau\left(\left(x-z_{n}\right) \gamma_{-t}^{E^{\times}}(y)\right)\right| \\
& \leqslant\left\|x-z_{n}\right\|_{E(\mathcal{M}, \tau)}\left\|\gamma_{-t}^{E^{\times}}(y)\right\|_{E(\mathcal{M}, \tau)^{\times}} \\
& =\left\|x-z_{n}\right\|_{E(\mathcal{M}, \tau)}\|y\|_{E(\mathcal{M}, \tau)^{\times}} \\
& \leqslant \frac{1}{n}\|y\|_{E(\mathcal{M}, \tau)^{\times}} .
\end{aligned}
$$

From the first part of the proof we know that the functions $f_{z_{n}}$ are continuous. Consequently, $f_{x}$ is a uniform limit of continuous functions and hence, $f_{x}$ itself is continuous. This completes the proof.

Let $\gamma$ be an $\mathbb{R}$-flow on $(\mathcal{M}, \tau)$ and $E$ be a separable r.i. BFS. By Corollary 4.1, the induced group $\gamma^{E}$ in $E(\mathcal{M}, \tau)$ is strongly continuous. In other words, $\gamma^{E}$ is a $C_{0}$-group of isometries in $E(\mathcal{M}, \tau)$. The infinitesimal generator of $\gamma^{E}$ is denoted by $\delta^{E}$ (and if $E=L_{p}$, we denote $\delta^{E}$ simply by $\left.\delta^{p}\right)$. Recall that this generator $\delta^{E}: \operatorname{Dom}\left(\delta^{E}\right) \rightarrow E(\mathcal{M}, \tau)$ is given by

$$
\delta^{E}(x)=\lim _{t \rightarrow 0} \frac{\gamma_{t}^{E}(x)-x}{t}, \quad x \in \operatorname{Dom}\left(\delta^{E}\right),
$$

where $\operatorname{Dom}\left(\delta^{E}\right)$ consists of those elements $x \in E(\mathcal{M}, \tau)$ for which this limit exists with respect to the norm in $E(\mathcal{M}, \tau)$. The operator $\delta^{E}$ is closed and $\operatorname{Dom}\left(\delta^{E}\right)$ is norm dense in $E(\mathcal{M}, \tau)$ (see, for example, [11]). Since $\gamma^{E}$ is a bounded group, the spectrum $\sigma\left(\delta^{E}\right)$ of $\delta^{E}$ is contained in $i \mathbb{R}$. For $\lambda \in \mathbb{C}$ with $\operatorname{Re} \lambda>0$, the resolvent operator $R\left(\lambda, \delta^{E}\right)=\left(\lambda-\delta^{E}\right)^{-1}$ is given by

$$
R\left(\lambda, \delta^{E}\right) x=\int_{0}^{\infty} \mathrm{e}^{-\lambda t} \gamma_{t}^{E}(x) \mathrm{d} t, \quad x \in E(\mathcal{M}, \tau),
$$

as a norm convergent Bochner integral in $E(\mathcal{M}, \tau)$. For $\lambda \in \mathbb{C}$ with $\operatorname{Re} \lambda<0$ we have

$$
R\left(\lambda, \delta^{E}\right) x=-R\left(-\lambda,-\delta^{E}\right) x=-\int_{0}^{\infty} \mathrm{e}^{\lambda t} \gamma_{-t}^{E}(x) \mathrm{d} t, \quad x \in E(\mathcal{M}, \tau) .
$$

Next we shall show that $\operatorname{Dom}\left(\delta^{E}\right) \cap \mathcal{M}$ is sufficiently large to determine the generator $\delta^{E}$. For this purpose we first prove the following lemma.

Lemma 4.2. If $x \in E(\mathcal{M}, \tau) \cap \mathcal{M}$ and $0<\lambda \in \mathbb{R}$, then $R\left(\lambda, \delta^{E}\right) x \in \operatorname{Dom}\left(\delta^{E}\right) \cap \mathcal{M}$ and $\delta^{E}\left(R\left(\lambda, \delta^{E}\right) x\right) \in E(\mathcal{M}, \tau) \cap \mathcal{M}$. 
Proof. Let $0<\lambda \in \mathbb{R}$ be given. It is clear that $R\left(\lambda, \delta^{E}\right) x \in \operatorname{Dom}\left(\delta^{E}\right)$ for all $x \in$ $E(\mathcal{M}, \tau) \cap \mathcal{M}$. To show that $R\left(\lambda, \delta^{E}\right) x \in \mathcal{M}$, take $y \in L_{1}(\mathcal{M}, \tau) \cap \mathcal{M}$. Using (4.3), (4.2) and (4.4) we find via trace duality that

$$
\begin{aligned}
\left\langle R\left(\lambda, \delta^{E}\right) x, y\right\rangle & =\int_{0}^{\infty} \mathrm{e}^{-\lambda t}\left\langle\gamma_{t}^{E}(x), y\right\rangle \mathrm{d} t \\
& =\int_{0}^{\infty} \mathrm{e}^{-\lambda t}\left\langle x, \gamma_{-t}^{1}(y)\right\rangle \mathrm{d} t \\
& =\left\langle x, \int_{0}^{\infty} \mathrm{e}^{-\lambda t} \gamma_{-t}^{1}(y) \mathrm{d} t\right\rangle \\
& =\left\langle x, R\left(\lambda,-\delta^{1}\right) y\right\rangle \\
& =\left\langle R\left(\lambda,-\delta^{1}\right)^{*} x, y\right\rangle .
\end{aligned}
$$

Since $L_{1}(\mathcal{M}, \tau) \cap \mathcal{M}$ separates the points of $L_{1}(\mathcal{M}, \tau)+\mathcal{M}$, we may conclude that $R\left(\lambda, \delta^{E}\right) x=R\left(\lambda,-\delta^{1}\right)^{*} x \in \mathcal{M}$, as $R\left(\lambda,-\delta^{1}\right)^{*}$ is a bounded linear operator from $\mathcal{M}$ into itself. This shows that $R\left(\lambda, \delta^{E}\right) x \in \operatorname{Dom}\left(\delta^{E}\right) \cap \mathcal{M}$ whenever $x \in E(\mathcal{M}, \tau) \cap \mathcal{M}$. Finally, for all $x \in E(\mathcal{M}, \tau) \cap \mathcal{M}$ we have $\delta^{E}\left(R\left(\lambda, \delta^{E}\right) x\right)=\lambda R\left(\lambda, \delta^{E}\right) x-x \in E(\mathcal{M}, \tau) \cap \mathcal{M}$.

Recall that a linear subspace $\mathcal{D}$ of $\operatorname{Dom}\left(\delta^{E}\right)$ is called a core for the operator $\delta^{E}$ if $\delta^{E}$ is the closure of the restriction of $\delta^{E}$ to $\mathcal{D}$ (equivalently, for every $x \in \operatorname{Dom}\left(\delta^{E}\right)$ there exists a sequence $\left\{x_{n}\right\}_{n=1}^{\infty}$ in $\mathcal{D}$ such that $x_{n} \rightarrow x$ and $\delta^{E}\left(x_{n}\right) \rightarrow \delta^{E}(x)$ as $\left.n \rightarrow \infty\right)$. If $\mathcal{D}$ is a linear subspace of $\operatorname{Dom}\left(\delta^{E}\right)$ that is dense in $E(\mathcal{M}, \tau)$ and invariant for the group $\gamma^{E}$, then $\mathcal{D}$ is a core for $\delta^{E}$ (see, for example, [11, Theorem 1.9]).

Theorem 4.3. Let $\gamma$ be an $\mathbb{R}$-flow on $(\mathcal{M}, \tau)$ and let $E$ be a separable r.i. BFS on $(0, \infty)$. If we define

$$
F=\left\{x \in \operatorname{Dom}\left(\delta^{E}\right) \cap \mathcal{M}: \delta^{E}(x) \in \mathcal{M}\right\},
$$

then $F$ is a core for the generator $\delta^{E}$ of $\gamma^{E}$.

Proof. If $x \in \operatorname{Dom}\left(\delta^{E}\right) \cap \mathcal{M}$ and $\delta^{E}(x) \in \mathcal{M}$, then $\gamma_{t}^{E}(x) \in \operatorname{Dom}\left(\delta^{E}\right)$ (see, for example, [11, Lemma 1.1]) and $\gamma_{t}^{E}(x)=\gamma_{t}(x) \in \mathcal{M}$. Moreover, $\delta^{E}\left(\gamma_{t}^{E}(x)\right)=\gamma_{t}^{E}\left(\delta^{E}(x)\right)=$ $\gamma_{t}\left(\delta^{E}(x)\right) \in \mathcal{M}$ for all $t \in \mathbb{R}$. This shows that $F$ is invariant for $\gamma^{E}$. It remains to show that $F$ is dense in $E(\mathcal{M}, \tau)$. Since $E$ has order-continuous norm, $L_{1}(\mathcal{M}, \tau) \cap \mathcal{M}$ is dense in $E(\mathcal{M}, \tau)$ and hence $E(\mathcal{M}, \tau) \cap \mathcal{M}$ is dense in $E(\mathcal{M}, \tau)$. Therefore, it is sufficient to show that $F$ is dense in $E(\mathcal{M}, \tau) \cap \mathcal{M}$. Let $x \in E(\mathcal{M}, \tau) \cap \mathcal{M}$ be given. It follows from Lemma 4.2 that $R\left(\lambda, \delta^{E}\right) x \in F$ for all $0<\lambda \in \mathbb{R}$. Furthermore, it is well known (see, for example, [29, Lemma 1.3.2]) that $\lim _{0<\lambda \rightarrow \infty} \lambda R\left(\lambda, \delta^{E}\right) x=x$ with respect to the norm in $E(\mathcal{M}, \tau)$. Hence, $F$ is dense in $E(\mathcal{M}, \tau) \cap \mathcal{M}$. Consequently, $F$ is dense in $E(\mathcal{M}, \tau)$ and invariant for $\gamma^{E}$, and so, by [11, Theorem 1.9], we may conclude that $F$ is a core for $\delta^{E}$.

Note that it follows in particular from the above theorem that $\operatorname{Dom}\left(\delta^{E}\right) \cap \mathcal{M}$ is a core for the generator $\delta^{E}$. The next proposition shows that $\delta^{E}$ acts as a derivation on $\operatorname{Dom}\left(\delta^{E}\right) \cap \mathcal{M}$. In the proof we will use the following result. 
Lemma 4.4. Let $E$ be a separable r.i. BFS on $(0, \infty)$. If $\left\{x_{n}\right\}_{n=1}^{\infty}$ is a sequence in $E(\mathcal{M}, \tau) \cap \mathcal{M}$ such that $\left\|x_{n}\right\|_{E(\mathcal{M}, \tau)} \rightarrow 0$ as $n \rightarrow \infty$ and $\sup _{n}\left\|x_{n}\right\|_{\infty}<\infty$, then $\left\|x_{n} y_{n}\right\|_{E(\mathcal{M}, \tau)} \rightarrow 0$ as $n \rightarrow \infty$ for any norm convergent sequence $\left\{y_{n}\right\}_{n=1}^{\infty}$ in $E(\mathcal{M}, \tau)$.

Proof. Let $C=\sup _{n}\left\|x_{n}\right\|_{\infty}$ and let $y \in E$ be such that $\left\|y_{n}-y\right\|_{E(\mathcal{M}, \tau)} \rightarrow 0$ as $n \rightarrow \infty$. Write $x_{n} y_{n}=x_{n}\left(y_{n}-y\right)+x_{n} y$. Since

$$
\left\|x_{n}\left(y_{n}-y\right)\right\|_{E(\mathcal{M}, \tau)} \leqslant\left\|x_{n}\right\|_{\infty}\left\|y_{n}-y\right\|_{E(\mathcal{M}, \tau)} \leqslant C\left\|y_{n}-y\right\|_{E(\mathcal{M}, \tau)}
$$

for all $n$, it is clear that $\left\|x_{n}\left(y_{n}-y\right)\right\|_{E(\mathcal{M}, \tau)} \rightarrow 0$ as $n \rightarrow \infty$. Furthermore, $\mu\left(x_{n} y\right) \leqslant$ $\left\|x_{n}\right\|_{\infty} \mu(y) \leqslant C \mu(y)$ for all $n$ (see [18, Lemma 2.5]) and $x_{n} y \rightarrow 0$ in measure (since the inclusion of $E(\mathcal{M}, \tau)$ into $\tilde{\mathcal{M}}$ is continuous; see, for example, [15, Lemma 4.4]). This implies that $\mu_{s}\left(x_{n} y\right) \rightarrow 0$ for all $s>0$ as $n \rightarrow \infty$. Defining $f_{n}(s)=\sup _{k \geqslant n} \mu_{s}\left(x_{k} y\right)$, we have $C \mu_{s}(y) \geqslant f_{n}(s) \downarrow_{n} 0$ for all $s>0$. The order continuity of the norm in $E$ implies that $\left\|f_{n}\right\| \downarrow 0$. Since $\left\|x_{n} y\right\|_{E(\mathcal{M}, \tau)}=\left\|\mu\left(x_{n} y\right)\right\|_{E} \leqslant\left\|f_{n}\right\|_{E}$ for all $n$, we may conclude that $\left\|x_{n} y\right\|_{E(\mathcal{M}, \tau)} \rightarrow 0$ as $n \rightarrow \infty$. This completes the proof of the lemma.

Proposition 4.5. Under the same assumptions as in Theorem 4.3, the subspace $\operatorname{Dom}\left(\delta^{E}\right) \cap \mathcal{M}$ is a subalgebra of $\mathcal{M}$ and $\delta^{E}(x y)=x \delta^{E}(y)+\delta^{E}(x) y$ for all $x, y \in$ $\operatorname{Dom}\left(\delta^{E}\right) \cap \mathcal{M}$.

Proof. Given $x, y \in \operatorname{Dom}\left(\delta^{E}\right) \cap \mathcal{M}$, it is clear that $x y \in E(\mathcal{M}, \tau) \cap \mathcal{M}$. We write

$$
\begin{aligned}
\frac{\gamma_{t}^{E}(x y)-x y}{t} & =\frac{\gamma_{t}^{E}(x) \gamma_{t}^{E}(y)-x y}{t} \\
& =\left(\gamma_{t}^{E}(x)-x\right) \frac{\gamma_{t}^{E}(y)-y}{t}+x \frac{\gamma_{t}^{E}(y)-y}{t}+\frac{\gamma_{t}^{E}(x)-x}{t} y .
\end{aligned}
$$

Since

$$
\lim _{t \rightarrow 0} \frac{\gamma_{t}^{E}(y)-y}{t}=\delta^{E}(y)
$$

with respect to the norm in $E(\mathcal{M}, \tau)$ and $x \in \mathcal{M}$, it follows that

$$
\lim _{t \rightarrow 0} x \frac{\gamma_{t}^{E}(y)-y}{t}=x \delta^{E}(y)
$$

with respect to the norm in $E(\mathcal{M}, \tau)$. Similarly, we see that

$$
\lim _{t \rightarrow 0} \frac{\left(\gamma_{t}^{E}(x)-x\right) y}{t}=\delta^{E}(x) y .
$$

Moreover, since $\left\|\gamma_{t}^{E}(x)-x\right\|_{\infty} \leqslant 2\|x\|_{\infty}$ for all $t \in \mathbb{R}$, it is an immediate consequence of Lemma 4.4 that

$$
\lim _{t \rightarrow 0}\left(\gamma_{t}^{E}(x)-x\right) \frac{\gamma_{t}^{E}(y)-y}{t}=0
$$

with respect to $\|\cdot\|_{E(\mathcal{M}, \tau)}$. Hence, we may conclude that $x y \in \operatorname{Dom}\left(\delta^{E}\right) \cap \mathcal{M}$ and $\delta^{E}(x y)=x \delta^{E}(y)+\delta^{E}(x) y$. 


\section{Double operator integrals and $\operatorname{Dom}\left(\delta^{E}\right)$}

We assume that $\gamma=\left\{\gamma_{t}\right\}_{t \in \mathbb{R}}$ is an $\mathbb{R}$-flow in $(\mathcal{M}, \tau)$ and that $E$ is a separable r.i. BFS on $(0, \infty)$. As in the previous section, $\delta^{E}$ denotes the infinitesimal generator of the induced $C_{0}$-group $\gamma^{E}=\left\{\gamma_{t}^{E}\right\}_{t \in \mathbb{R}}$ on the corresponding symmetric operator space $E(\mathcal{M}, \tau)$. In this section we are particularly interested in the following question.

For which functions $f: \mathbb{R} \rightarrow \mathbb{C}$ is $f(x) \in \operatorname{Dom}\left(\delta^{E}\right)$ whenever $x^{*}=x \in \operatorname{Dom}\left(\delta^{E}\right)$ ?

Some additional conditions on the function $f$ are inevitable. In particular, to guarantee that $f(x) \in E(\mathcal{M}, \tau)$ whenever $x^{*}=x \in E(\mathcal{M}, \tau)$, we shall assume that $|f(\lambda)| \leqslant A|\lambda|$ for all $\lambda \in \mathbb{R}$ and some constant $0 \leqslant A \in \mathbb{R}$. Indeed, under this assumption we have $|f(x)| \leqslant A|x|$ for all $x^{*}=x \in E(\mathcal{M}, \tau)$, and so $f(x) \in E(\mathcal{M}, \tau)$. In this situation, it follows from Proposition 3.3 (i) that

$$
\gamma_{t}^{E}(f(x))=f\left(\gamma_{t}^{E}(x)\right), \quad t \in \mathbb{R} .
$$

Assuming, moreover, that $f$ is continuously differentiable and that $x \in \operatorname{Dom}\left(\delta^{E}\right)$, formal differentiation of (5.1) yields $f(x) \in \operatorname{Dom}\left(\delta^{E}\right)$ and

$$
\delta^{E}(f(x))=f^{\prime}(x) \delta^{E}(x) .
$$

In some special situations, e.g. if $\delta^{E}$ is bounded and the elements $x$ and $\delta^{E}(x)$ commute, (5.2) can indeed be justified (see, for example, [28, pp. 598, 599] or [8, p. 236]). However, as we will see is this section, in the general setting considered in this paper an appropriate modified version of (5.2) is valid, where the right-hand side of this equality uses the double operator integral theory, recently developed in this setting in $[\mathbf{1 2}, \mathbf{1 3}]$ (see also $[\mathbf{1}, \mathbf{3}-\mathbf{5}]$ in the setting of symmetrically normed ideals of compact operators). To formulate and prove our results, we first recall the necessary definitions and theorems concerning double operator integrals. For the details we refer the reader to $[\mathbf{1 2}, \mathbf{1 3}]$.

In [13] the theory of double operator integrals has been developed in general non-commutative symmetric spaces. In the present paper we will only consider non-commutative spaces $E(\mathcal{M}, \tau)$, where $E$ is a separable r.i. BFS on $(0, \infty)$, so $E(\mathcal{M}, \tau)$ has ordercontinuous norm. In this situation a simpler and less technical version of the general theory can be used (see also [12]). Suppose that $a$ and $b$ are self-adjoint operators in $\mathcal{H}$ affiliated with $\mathcal{M}$. For every $B \in \operatorname{Bor}(\mathbb{R})$, define the projections

$$
P_{E}^{a}(B), Q_{E}^{b}(B): E(\mathcal{M}, \tau) \rightarrow E(\mathcal{M}, \tau)
$$

by

$$
P_{E}^{a}(B) x=e^{a}(B) x, \quad Q_{E}^{b}(B) x=x e^{b}(B), \quad x \in E(\mathcal{M}, \tau) .
$$

Evidently, $P_{E}^{a}, Q_{E}^{b}: \operatorname{Bor}(\mathbb{R}) \rightarrow \mathcal{L}(E(\mathcal{M}, \tau))$ are two commuting (countably additive) spectral measures (in the sense of $[\mathbf{1 7}$, Chapter $\mathrm{X}]$ ) satisfying

$$
\left\|P_{E}^{a}(B)\right\|_{\mathcal{L}(E(\mathcal{M}, \tau))} \leqslant 1 \quad \text { and } \quad\left\|Q_{E}^{b}(B)\right\|_{\mathcal{L}(E(\mathcal{M}, \tau))} \leqslant 1 \quad \text { for all } B \in \operatorname{Bor}(\mathbb{R})
$$


We denote by $\mathcal{A}$ the algebra of subsets of $\mathbb{R}^{2}$ which is generated by all Borel rectangles $A \times B$ with $A, B \in \operatorname{Bor}(\mathbb{R})$. Let $P_{E}^{a} \otimes Q_{E}^{b}: \mathcal{A} \rightarrow \mathcal{L}(E)$ be the product measure, that is,

$$
P_{E}^{a} \otimes Q_{E}^{b}(A \times B)=P_{E}^{a}(A) Q_{E}^{b}(B)
$$

for all $A, B \in \operatorname{Bor}(\mathbb{R})$. It is easily verified that $P_{E}^{a} \otimes Q_{E}^{b}$ is a (finitely additive) spectral measure. First consider the case that $E=L_{2}(0, \infty)$, that is, $E(\mathcal{M}, \tau)=L_{2}(\mathcal{M}, \tau)$. In this situation $P_{L_{2}}^{a}$ and $Q_{L_{2}}^{b}$ take their values in the orthogonal projections of the Hilbert space $L_{2}(\mathcal{M}, \tau)$. As is well known (see, for example, [6, Theorem V.2.6]), the product measure $P_{L_{2}}^{a} \otimes Q_{L_{2}}^{b}$ extends uniquely to a countably additive spectral measure on the Borel sets Bor $\left(\mathbb{R}^{2}\right)$, taking its values in the orthogonal projections of $L_{2}(\mathcal{M}, \tau)$. This extension is also denoted by $P_{L_{2}}^{a} \otimes Q_{L_{2}}^{b}$, so

$$
P_{L_{2}}^{a} \otimes Q_{L_{2}}^{b}: \operatorname{Bor}\left(\mathbb{R}^{2}\right) \rightarrow \mathcal{L}\left(L_{2}(\mathcal{M}, \tau)\right) .
$$

For every bounded Borel function $\varphi \in B\left(\mathbb{R}^{2}\right)$, the spectral integral

$$
T_{\varphi, 2}^{a, b}=\int_{\mathbb{R}^{2}} \varphi \mathrm{d}\left(P_{L_{2}}^{a} \otimes Q_{L_{2}}^{b}\right)
$$

is a bounded linear operator on $L_{2}(\mathcal{M}, \tau)$ and the map $\varphi \mapsto T_{\varphi, 2}^{a, b}$ is an algebra homomorphism from the algebra $B\left(\mathbb{R}^{2}\right)$ of all complex-valued bounded Borel functions into $\mathcal{L}\left(L_{2}(\mathcal{M}, \tau)\right)$.

Now we return to the situation that $E$ is an arbitrary separable r.i. BFS on $(0, \infty)$. Note that in this case $E(\mathcal{M}, \tau) \cap L_{2}(\mathcal{M}, \tau)$ is a dense subspace of $E(\mathcal{M}, \tau)$.

Definition 5.1. A function $\varphi \in B\left(\mathbb{R}^{2}\right)$ is said to be $P_{E}^{a} \otimes Q_{E}^{b}$-integrable if

$$
T_{\varphi, 2}^{a, b}\left(E(\mathcal{M}, \tau) \cap L_{2}(\mathcal{M}, \tau)\right) \subseteq E(\mathcal{M}, \tau) \cap L_{2}(\mathcal{M}, \tau)
$$

and the restriction of $T_{\varphi, 2}^{a, b}$ to $E(\mathcal{M}, \tau) \cap L_{2}(\mathcal{M}, \tau)$ is continuous with respect to $\|\cdot\|_{E(\mathcal{M}, \tau)}$. If this is the case, then the restriction $\left.\left(T_{\varphi, 2}^{a, b}\right)\right|_{E(\mathcal{M}, \tau) \cap L_{2}(\mathcal{M}, \tau)}$ has a unique extension to a bounded linear operator on $E(\mathcal{M}, \tau)$, which is denoted by

$$
T_{\varphi, E}^{a, b}=\int_{\mathbb{R}^{2}} \varphi \mathrm{d}\left(P_{E}^{a} \otimes Q_{E}^{b}\right) .
$$

The collection of all $P_{E}^{a} \otimes Q_{E}^{b}$-integrable functions is denoted by $\mathfrak{J}_{E}\left(P_{E}^{a} \otimes Q_{E}^{b}\right)$.

The set $\mathfrak{J}_{E}\left(P_{E}^{a} \otimes Q_{E}^{b}\right)$ is a subalgebra of $B\left(\mathbb{R}^{2}\right)$ and the map $\varphi \mapsto T_{\varphi, E}^{a, b}$ is an algebra homomorphism from $\mathfrak{J}_{E}\left(P_{E}^{a} \otimes Q_{E}^{b}\right)$ into $\mathcal{L}(E(\mathcal{M}, \tau))$. If $F$ is another separable r.i. BFS on $(0, \infty)$, then $E(\mathcal{M}, \tau) \cap F(\mathcal{M}, \tau) \cap L_{2}(\mathcal{M}, \tau)$ is dense in both $E(\mathcal{M}, \tau)$ and $F(\mathcal{M}, \tau)$. Moreover, if

$$
\varphi \in \mathfrak{J}_{E}\left(P_{E}^{a} \otimes Q_{E}^{b}\right) \cap \mathfrak{J}_{F}\left(P_{F}^{a} \otimes Q_{F}^{b}\right),
$$

then $T_{\varphi, E}^{a, b}$ and $T_{\varphi, F}^{a, b}$ agree on $E(\mathcal{M}, \tau) \cap F(\mathcal{M}, \tau) \cap L_{2}(\mathcal{M}, \tau)$, and hence on $E(\mathcal{M}, \tau) \cap$ $F(\mathcal{M}, \tau)$. Therefore, there is no danger of confusion if we denote the operator $T_{\varphi, E}^{a, b}$ simply by $T_{\varphi}^{a, b}$, as we will do from now on.

Next we will exhibit a large class of functions in $B\left(\mathbb{R}^{2}\right)$ which is contained in $\mathfrak{J}_{E}\left(P_{E}^{a} \otimes\right.$ $\left.Q_{E}^{b}\right)$ for any separable r.i. BFS $E$. 
Definition 5.2. We denote by $\mathfrak{A}_{0}$ the collection of all functions $\varphi \in B\left(\mathbb{R}^{2}\right)$ for which there exist a $\sigma$-finite measure space $(S, \Sigma, \nu)$ and two complex-valued measurable functions $\alpha$ and $\beta$ on the product measurable space $(\mathbb{R} \times S$, Bor $(\mathbb{R}) \otimes \Sigma)$, satisfying $\alpha(\cdot, s), \beta(\cdot, s) \in B(\mathbb{R})$ for all $s \in S$ and

$$
C_{\alpha, \beta}=\int_{S}\|\alpha(\cdot, s)\|_{\infty}\|\beta(\cdot, s)\|_{\infty} \mathrm{d} \nu(s)<\infty
$$

such that

$$
\varphi(\lambda, \mu)=\int_{S} \alpha(\lambda, s) \beta(\mu, s) \mathrm{d} \nu(s), \quad(\lambda, \mu) \in \mathbb{R}^{2} .
$$

For every $\varphi \in \mathfrak{A}_{0}$ we define $\|\varphi\|_{\mathfrak{A}_{0}}=\inf C_{\alpha, \beta}$, where the infimum is taken over all possible representations (5.3) of $\varphi$. Furthermore, we denote by $\mathfrak{C}_{0}$ the collection of all $\varphi \in \mathfrak{A}_{0}$ for which the functions $\alpha$ and $\beta$ in (5.3) can be chosen such that $\alpha(\cdot, s), \beta(\cdot, s) \in C_{b}(\mathbb{R})$ (where $C_{b}(\mathbb{R})$ denotes the algebra of all bounded continuous complex-valued functions on $\mathbb{R})$.

For the convenience of the reader, we collect in the next proposition some of the results of $[\mathbf{1 2}]$ which will be used in the present section.

Proposition 5.3. In the above setting the following statements hold.

(i) $\left(\mathfrak{A}_{0},\|\cdot\|_{\mathfrak{A}_{0}}\right)$ is a Banach algebra and $\|\varphi\|_{\infty} \leqslant\|\varphi\|_{\mathfrak{A}_{0}}$ for all $\varphi \in \mathfrak{A}_{0}$. Moreover, $\mathfrak{C}_{0}$ is a closed subalgebra of $\mathfrak{A}_{0}$ consisting of continuous functions [12, Lemma 4.6].

(ii) If $a$ and $b$ are self-adjoint operators, affiliated with $\mathcal{M}$, then $\mathfrak{A}_{0} \subseteq \mathfrak{J}_{E}\left(P_{E}^{a} \otimes Q_{E}^{b}\right)$ and the map $\varphi \mapsto T_{\varphi}^{a, b}$ is an algebra homomorphism from $\mathfrak{A}_{0}$ into $\mathcal{L}(E)$ satisfying $\left\|T_{\varphi}^{a, b}\right\|_{\mathcal{L}(E)} \leqslant\|\varphi\|_{\mathfrak{A}_{0}}[\mathbf{1 2}$, Proposition 4.7].

(iii) If $\left\{a_{n}\right\}_{n=1}^{\infty}$ and $\left\{b_{n}\right\}_{n=1}^{\infty}$ converge in $\tilde{\mathcal{M}}_{h}$ in measure to $a, b \in \tilde{\mathcal{M}}_{h}$, respectively, then $\left\|T_{\varphi}^{a_{n}, b_{n}} x-T_{\varphi}^{a, b} x\right\|_{E(\mathcal{M}, \tau)} \rightarrow 0$ as $n \rightarrow \infty$ for all $x \in E(\mathcal{M}, \tau)$ and $\varphi \in \mathfrak{C}_{0}[\mathbf{1 2}$, Lemma 5.14].

Suppose that $F$ is a symmetric (not necessarily separable) BFS on $(0, \infty)$. The natural embedding of the space $F(\mathcal{M}, \tau)$ in its Köthe bidual $F(\mathcal{M}, \tau)^{\times \times}$is contractive but not isometric, in general. In the next lemma we need the condition that this embedding is an isometry. This condition is equivalent to the lower semi-continuity of the norm in $F(\mathcal{M}, \tau)$, that is, $0 \leqslant x_{\alpha} \uparrow x$ in $F(\mathcal{M}, \tau)$ implies that $\|x\|_{F(\mathcal{M}, \tau)}=\sup _{\alpha}\left\|x_{\alpha}\right\|_{F(\mathcal{M}, \tau)}$ (see [16, Proposition 5.14]). If the norm in the BFS $F$ is lower semi-continuous (also called a Fatou norm), then so is the norm in the non-commutative space $F(\mathcal{M}, \tau)$ (as follows easily from [16, Lemma 1.6]). Note furthermore that if $F$ has an order-continuous norm (equivalently, is separable), then the norm in $F$ is lower semi-continuous.

Lemma 5.4. Suppose that $F$ is a symmetric BFS with lower semi-continuous norm. If $a$ and $b$ are self-adjoint operators affiliated with $\mathcal{M}$ and if $\varphi \in \mathfrak{A}_{0}$, then $\left\|T_{\varphi}^{a, b}(x)\right\|_{F(\mathcal{M}, \tau)} \leqslant\|\varphi\|_{\mathfrak{A}_{0}}\|x\|_{F}$ for all $x \in L_{1}(\mathcal{M}, \tau) \cap \mathcal{M}$. 
Proof. In the proof of [12, Proposition 4.7], it is shown that $T_{\varphi}^{a, b}(x) \in L_{1}(\mathcal{M}, \tau) \cap \mathcal{M}$ whenever $x \in L_{1}(\mathcal{M}, \tau) \cap \mathcal{M}$. We use a similar argument to prove the statement of the present lemma. Represent the function $\varphi$ as in (5.3). For $s \in S$, define the operator $T_{s} \in \mathcal{L}\left(L_{2}(\mathcal{M}, \tau)\right)$ by

$$
T_{s}(x)=\alpha(a, s) x \beta(b, s), \quad x \in L_{2}(\mathcal{M}, \tau) .
$$

Note that (5.4) also defines a bounded linear operator on $F(\mathcal{M}, \tau)$, satisfying

$$
\left\|T_{s}(x)\right\|_{F(\mathcal{M}, \tau)} \leqslant\|\alpha(\cdot, s)\|_{\infty}\|\beta(\cdot, s)\|_{\infty}\|x\|_{F(\mathcal{M}, \tau)}, \quad x \in F(\mathcal{M}, \tau) .
$$

In the proof of $[\mathbf{1 2}$, Proposition 4.7], it is shown that

$$
\left\langle T_{\varphi}^{a, b} x, y\right\rangle=\int_{S}\left\langle T_{s} x, y\right\rangle \mathrm{d} \nu(s), \quad x, y \in L_{2}(\mathcal{M}, \tau)
$$

(here $\langle\cdot, \cdot\rangle$ is the duality pairing given by (2.3)). Fix $x \in L_{1}(\mathcal{M}, \tau) \cap \mathcal{M}$ and take $y \in L_{1}(\mathcal{M}, \tau) \cap \mathcal{M}$ with $\|y\|_{F(\mathcal{M}, \tau) \times} \leqslant 1$. Let $T_{\varphi}^{a, b}(x) y=u\left|T_{\varphi}^{a, b}(x) y\right|$ be the polar decomposition of $T_{\varphi}^{a, b}(x) y$. Using (5.5) and (5.6) it follows that

$$
\begin{aligned}
\tau\left(\left|T_{\varphi}^{a, b}(x) y\right|\right) & =\tau\left(T_{\varphi}^{a, b}(x) y u^{*}\right) \\
& =\int_{S}\left\langle T_{s} x, y u^{*}\right\rangle \mathrm{d} \nu(s) \\
& \leqslant\left(\int_{S}\|\alpha(\cdot, s)\|_{\infty}\|\beta(\cdot, s)\|_{\infty} \mathrm{d} \nu(s)\right)\|x\|_{F(\mathcal{M}, \tau)}\|y\|_{F(\mathcal{M}, \tau)^{\times}} \\
& \leqslant C_{\alpha, \beta}\|x\|_{F(\mathcal{M}, \tau)} .
\end{aligned}
$$

Via [16, Proposition 5.3], and by the assumption on the norm of $F$, we may conclude that $\left\|T_{\varphi}^{a, b}(x)\right\|_{F(\mathcal{M}, \tau)}=\left\|T_{\varphi}^{a, b}(x)\right\|_{F(\mathcal{M}, \tau) \times \times} \leqslant C_{\alpha, \beta}\|x\|_{F(\mathcal{M}, \tau)}$. The result of the lemma is now clear from the definition of the norm in $\mathfrak{A}_{0}$.

Given a symmetric BFS $E$, the space $E \cap L_{\infty}(0, \infty)$, equipped with the norm given by

$$
\|f\|_{E \cap L_{\infty}(0, \infty)}=\max \left(\|f\|_{E},\|f\|_{L_{\infty}(0, \infty)}\right),
$$

is also a symmetric BFS (but $E \cap L_{\infty}(0, \infty)$ is not separable, even if $E$ is). Moreover, it is easy to see that

$$
E(\mathcal{M}, \tau) \cap L_{\infty}(\mathcal{M}, \tau)=\left(E \cap L_{\infty}\right)(\mathcal{M}, \tau)
$$

Lemma 5.5. Let $E$ be a separable r.i. BFS and suppose that $a$ and $b$ are selfadjoint operators affiliated with $(\mathcal{M}, \tau)$. If $\varphi \in \mathfrak{A}_{0}$, then $T_{\varphi}^{a, b}(x) \in E(\mathcal{M}, \tau) \cap \mathcal{M}$ and $\left\|T_{\varphi}^{a, b}(x)\right\|_{E(\mathcal{M}, \tau) \cap \mathcal{M}} \leqslant\|\varphi\|_{\mathfrak{A}_{0}}\|x\|_{E(\mathcal{M}, \tau) \cap \mathcal{M}}$ for all $x \in E(\mathcal{M}, \tau) \cap \mathcal{M}$.

Proof. Since $E$ has order-continuous norm, it is clear that the norm of $E \cap L_{\infty}$ is lower semi-continuous. Therefore, applying Lemma 5.4 to $F=E \cap L_{\infty}$, it follows that

$$
\left\|T_{\varphi}^{a, b}(x)\right\|_{E(\mathcal{M}, \tau) \cap \mathcal{M}} \leqslant\|\varphi\|_{\mathfrak{A}_{0}}\|x\|_{E(\mathcal{M}, \tau) \cap \mathcal{M},}, \quad x \in L_{1}(\mathcal{M}, \tau) \cap \mathcal{M} .
$$




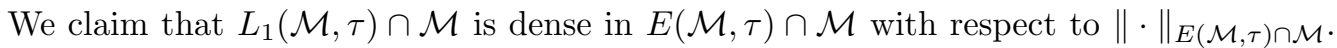
Indeed, let $0 \leqslant a \in E(\mathcal{M}, \tau) \cap \mathcal{M}$ be given and define $a_{n}=a e^{a}(1 / n, \infty)$ for all $n \in \mathbb{N}$. It is evident that $a_{n} \in \mathcal{M}$ and, since $E(\mathcal{M}, \tau)$ has order-continuous norm, we also have $\tau\left(e^{a}(1 / n, \infty)\right)<\infty$ (see the comments at the end of $\S 2$ ), and so $a_{n} \in L_{1}(\mathcal{M}, \tau)$. Moreover, $0 \leqslant a-a_{n}=a e^{a}[0,1 / n]$, which implies that $0 \leqslant a-a_{n} \leqslant(1 / n) \mathbf{1}$ and so $a-a_{n} \downarrow 0$

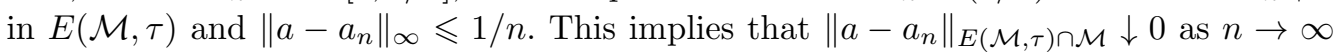
and the claim is proved.

Let $x \in E(\mathcal{M}, \tau) \cap \mathcal{M}$ be given and take a sequence $\left\{x_{n}\right\}_{n=1}^{\infty}$ in $L_{1}(\mathcal{M}, \tau) \cap \mathcal{M}$ such that $\left\|x-x_{n}\right\|_{E(\mathcal{M}, \tau) \cap \mathcal{M}} \rightarrow 0$ as $n \rightarrow \infty$. It follows from (5.7) that $\left\{T_{\varphi}^{a, b}\left(x_{n}\right)\right\}_{n=1}^{\infty}$ is a Cauchy sequence in $E(\mathcal{M}, \tau) \cap \mathcal{M}$, and so there exists $y \in E(\mathcal{M}, \tau) \cap \mathcal{M}$ such that $\left\|T_{\varphi}^{a, b}\left(x_{n}\right)-y\right\|_{E(\mathcal{M}, \tau) \cap \mathcal{M}} \rightarrow 0$ as $n \rightarrow \infty$. Moreover, since $T_{\varphi}^{a, b}$ is a bounded linear operator in $E(\mathcal{M}, \tau)$, we also have $\left\|T_{\varphi}^{a, b}\left(x_{n}\right)-T_{\varphi}^{a, b}(x)\right\|_{E(\mathcal{M}, \tau)} \rightarrow 0$ as $n \rightarrow \infty$. Consequently, $T_{\varphi}^{a, b}(x)=y \in E(\mathcal{M}, \tau) \cap \mathcal{M}$ and, from (5.7), applied to $x_{n}$ and taking the limit as $n \rightarrow \infty$, it follows that $\left\|T_{\varphi}^{a, b}(x)\right\|_{E(\mathcal{M}, \tau) \cap \mathcal{M}} \leqslant\|\varphi\|_{\mathfrak{A}_{0}}\|x\|_{E(\mathcal{M}, \tau) \cap \mathcal{M}}$.

For a Borel function $f: \mathbb{R} \rightarrow \mathbb{C}$ we denote by $\psi_{f}$ any Borel function $\psi_{f}: \mathbb{R}^{2} \rightarrow \mathbb{C}$ that satisfies

$$
\psi_{f}(\lambda, \mu)=\frac{f(\lambda)-f(\mu)}{\lambda-\mu}
$$

for all $(\lambda, \mu) \in \mathbb{R}^{2}$ with $\lambda \neq \mu$. Observe that $\psi_{f} \in B\left(\mathbb{R}^{2}\right)$ if and only if $f$ is a Lipschitz function on $\mathbb{R}$. Moreover, there exists at most one continuous function $\psi_{f}$ satisfying (5.8), which is the case if and only if $f \in C^{1}(\mathbb{R})$ (that is, $f$ is continuously differentiable). By the statement $\psi_{f} \in \mathfrak{A}_{0}\left(\right.$ or $\mathfrak{C}_{0}$ ) we mean that there exists a function $\psi_{f} \in \mathfrak{A}_{0}$ (or $\left.\mathfrak{C}_{0}\right)$ satisfying (5.8) for all $\lambda \neq \mu$. Furthermore, we denote by $\Psi^{-1}\left(\mathfrak{A}_{0}\right)$ the linear space of all Borel functions $f: \mathbb{R} \rightarrow \mathbb{C}$ such that $\psi_{f} \in \mathfrak{A}_{0}$. The space $\Psi^{-1}\left(\mathfrak{C}_{0}\right)$ is defined similarly. Note that all functions $f \in \Psi^{-1}\left(\mathfrak{C}_{0}\right)$ are continuously differentiable with a bounded derivative. Furthermore, observe that every $f \in \Psi^{-1}\left(\mathfrak{A}_{0}\right)$ with $f(0)=0$ satisfies $|f(\lambda)| \leqslant A|\lambda|$ for all $\lambda \in \mathbb{R}$ and some constant $0 \leqslant A \in \mathbb{R}$. We will need the following result (which is a special case of [12, Proposition 5.2]).

Proposition 5.6. Let $E$ be a separable r.i. BFS on $(0, \infty)$. If $f \in \Psi^{-1}\left(\mathfrak{C}_{0}\right)$ and $a, b \in \tilde{\mathcal{M}}_{h}$ such that $a-b \in E(\mathcal{M}, \tau)$, then $f(a)-f(b) \in E$ and $f(a)-f(b)=T_{\psi_{f}}^{a, b}(a-b)$.

Now we are in a position to prove the main result of this section.

Theorem 5.7. Let $\gamma=\left\{\gamma_{t}\right\}_{t \in \mathbb{R}}$ be an $\mathbb{R}$-flow in $(\mathcal{M}, \tau)$ and let $E$ be a separable r.i. BFS on $(0, \infty)$. If $a^{*}=a \in \operatorname{Dom}\left(\delta^{E}\right)$ and $f \in \Psi^{-1}\left(\mathfrak{C}_{0}\right)$ with $f(0)=0$, then $f(a) \in \operatorname{Dom}\left(\delta^{E}\right)$ and

$$
\delta^{E}(f(a))=T_{\psi_{f}}^{a, a}\left(\delta^{E}(a)\right)
$$

Proof. Let $a^{*}=a \in \operatorname{Dom}\left(\delta^{E}\right)$ be given and let $\left\{t_{n}\right\}_{n=1}^{\infty}$ be a sequence in $\mathbb{R}$ such that $t_{n} \neq 0$ for all $n$ and $t_{n} \rightarrow 0$ as $n \rightarrow \infty$. Writing $\gamma_{t_{n}}(a)=a+t_{n} \delta^{E}(a)+R_{n}$, we have $\left\|t_{n}^{-1} R_{n}\right\|_{E(\mathcal{M}, \tau)} \rightarrow 0$ as $n \rightarrow \infty$. For convenience, we also write $a_{n}=\gamma_{t_{n}}(a)$. Using 
Propositions 3.3 (i) and 5.6 we find that

$$
\begin{aligned}
\frac{\gamma_{t_{n}}(f(a))-f(a)}{t_{n}} & =\frac{f\left(\gamma_{t_{n}}(a)\right)-f(a)}{t_{n}} \\
& =\frac{f\left(a+t_{n} \delta^{E}(a)+R_{n}\right)-f(a)}{t_{n}} \\
& =\frac{1}{t_{n}} T_{\psi_{f}}^{a_{n}, a}\left(t_{n} \delta^{E}(a)+R_{n}\right) \\
& =T_{\psi_{f}}^{a_{n}, a}\left(\delta^{E}(a)\right)+T_{\psi_{f}}^{a_{n}, a}\left(\frac{R_{n}}{t_{n}}\right)
\end{aligned}
$$

for all $n$. It follows from Proposition 5.3 (ii) that

$$
\begin{aligned}
\left\|T_{\psi_{f}}^{a_{n}, a}\left(\frac{R_{n}}{t_{n}}\right)\right\|_{E(\mathcal{M}, \tau)} & \leqslant\left\|T_{\psi_{f}}^{a_{n}, a}\right\|_{\mathcal{L}(E(\mathcal{M}, \tau))}\left\|t_{n}^{-1} R_{n}\right\|_{E(\mathcal{M}, \tau)} \\
& \leqslant\left\|\psi_{f}\right\|_{\mathfrak{A}_{0}}\left\|t_{n}^{-1} R_{n}\right\|_{E(\mathcal{M}, \tau)} .
\end{aligned}
$$

This shows that $\left\|T_{\psi_{f}}^{a_{n}, a}\left(t_{n}^{-1} R_{n}\right)\right\|_{E(\mathcal{M}, \tau)} \rightarrow 0$ as $n \rightarrow \infty$. Moreover, since $a_{n}=\gamma_{t_{n}}(a) \rightarrow a$ in $E(\mathcal{M}, \tau)$, and, hence, in measure, Proposition 5.3 (iii) implies that

$$
T_{\psi_{f}}^{a_{n}, a}\left(\delta^{E}(a)\right) \rightarrow T_{\psi_{f}}^{a, a}\left(\delta^{E}(a)\right) \in E(\mathcal{M}, \tau) \quad \text { as } n \rightarrow \infty .
$$

Therefore, it follows that

$$
\lim _{n \rightarrow \infty} \frac{\gamma_{t_{n}}(f(a))-f(a)}{t_{n}}=T_{\psi_{f}}^{a, a}\left(\delta^{E}(a)\right)
$$

with respect to the norm of $E(\mathcal{M}, \tau)$. Since this holds for any such sequence $\left\{t_{n}\right\}_{n=1}^{\infty}$, we may conclude that $f(a) \in \operatorname{Dom}\left(\delta^{E}\right)$ and $\delta^{E}(f(a))=T_{\psi_{f}}^{a, a}\left(\delta^{E}(a)\right)$.

Remark 5.8. The proof of the preceding proposition is similar to that of $[\mathbf{1}$, Theorem 5.1] in the setting of symmetrically normed ideals of compact operators. However, the technical ingredients used in the proofs of Propositions 5.3 and 5.6 in the present general setting are distinct from the theory of Birman and Solomyak [5] employed in [1].

It was shown by Peller $[\mathbf{3 0}]$ that the Besov space $B_{\infty, 1}^{1}(\mathbb{R})$ is contained in $\Psi^{-1}\left(\mathfrak{C}_{0}\right)$. In the next corollary we point out two interesting consequences of this result in combination with Theorem 5.7. For the relevant details we refer the reader to $[\mathbf{1 2}, \S 7]$. We denote by $\mathcal{S}^{\prime}(\mathbb{R})$ the space of tempered distributions on $\mathbb{R}$ and, for $f \in \mathcal{S}^{\prime}(\mathbb{R})$, we denote its Fourier transform by $\mathcal{F} f$. The space of all bounded Borel measures of $\mathbb{R}$ is denoted by $M_{b}(\mathbb{R})$. Furthermore, given $\varepsilon>0, C^{1+\varepsilon}(\mathbb{R})$ is the space of all continuously differentiable functions on $\mathbb{R}$, with bounded derivative $f^{\prime}$ which satisfies $\left|f^{\prime}(\lambda)-f^{\prime}(\mu)\right| \leqslant C|\lambda-\mu|^{\varepsilon}$ for all $\lambda, \mu \in \mathbb{R}$ and some constant $0 \leqslant C \in \mathbb{R}$.

Corollary 5.9. The assertion of Theorem 5.7 holds for any function $f \in \mathcal{S}^{\prime}(\mathbb{R})$ with $f(0)=0$, satisfying one of the following conditions:

(i) $\mathcal{F} f^{\prime} \in M_{b}(\mathbb{R})$; or

(ii) $f \in C^{1+\varepsilon}(\mathbb{R})$ for some $\varepsilon>0$. 


\section{6. $\mathbb{R}$-flows induced by $\tau$-measurable operators}

In this section we consider $\mathbb{R}$-flows $\gamma=\left\{\gamma_{t}\right\}_{t \in \mathbb{R}}$ on $(\mathcal{M}, \tau)$ of the special form

$$
\gamma_{t}(x)=\mathrm{e}^{\mathrm{i} t a} x \mathrm{e}^{-\mathrm{i} t a}, \quad x \in \mathcal{M},
$$

where $a$ is a self-adjoint $\tau$-measurable operator. First we observe that (6.1) defines an $\mathbb{R}$-flow on $\mathcal{M}$ for any $a \in \tilde{\mathcal{M}}_{h}$. Indeed, $\left\{\mathrm{e}^{\mathrm{i} t a}\right\}_{t \in \mathbb{R}}$ is a strongly continuous unitary group on $\mathcal{H}$ which is contained in $\mathcal{M}$. Hence, each $\gamma_{t}$ is a trace preserving $*$-automorphism in $\mathcal{M}$. Furthermore, $\mathrm{e}^{\mathrm{i} t a} \rightarrow 0$ as $t \rightarrow 0$ with respect to the strong operator topology, and hence, since multiplication on norm-bounded subsets of $\mathcal{L}(\mathcal{H})$ is strongly continuous (see $[\mathbf{1 4}$, p. 33]), it follows that $\mathrm{e}^{\mathrm{i} t a} x \mathrm{e}^{-\mathrm{i} t a} \rightarrow 0$ strongly as $t \rightarrow 0$ for all $x \in \mathcal{M}$. Moreover, on normbounded subset of $\mathcal{L}(\mathcal{H})$ the strong and ultra-strong topology coincide (see [14, p. 37]), and so $\mathrm{e}^{\mathrm{i} t a} x \mathrm{e}^{-\mathrm{i} t a} \rightarrow 0$ ultra-strongly, and hence ultra-weakly, as $t \rightarrow 0$ for all $x \in \mathcal{M}$. This shows that $\left\{\gamma_{t}\right\}_{t \in \mathbb{R}}$ is an $\mathbb{R}$-flow on $\mathcal{M}$. It is easily verified that the extension $\tilde{\gamma}=\left\{\tilde{\gamma}_{t}\right\}_{t \in \mathbb{R}}$ to $\tilde{\mathcal{M}}$ (see 3.3 and the discussion at the beginning of $\S 4$ ) is also given by $\tilde{\gamma}_{t}(x)=\mathrm{e}^{\mathrm{i} t a} x \mathrm{e}^{-\mathrm{i} t a}$ for all $x \in \tilde{\mathcal{M}}$. Our objective in this section is to obtain a complete description of the infinitesimal generator $\delta^{E}$ of $\gamma^{E}$ (see Theorem 6.8). The proof of this result is divided into several lemmas. As usual, for $x, y \in \tilde{\mathcal{M}}$ we denote by $[x, y]$ the commutator, that is, $[x, y]=x y-y x$.

Lemma 6.1. Let $a \in \tilde{\mathcal{M}}_{h}$ and $\tilde{\gamma}_{t}: \tilde{\mathcal{M}} \rightarrow \tilde{\mathcal{M}}$ be defined by $\tilde{\gamma}_{t}(x)=\mathrm{e}^{\mathrm{i} t a} x \mathrm{e}^{-\mathrm{i} t a}$ for all $x \in \tilde{\mathcal{M}}$ and all $t \in \mathbb{R}$. Then

$$
\lim _{t \rightarrow 0} \frac{\tilde{\gamma}_{t}(x)-x}{t}=\mathrm{i}[a, x]
$$

with respect to the measure topology for all $x \in \tilde{\mathcal{M}}$.

Proof. Given $x \in \tilde{\mathcal{M}}$, we write

$$
\frac{\tilde{\gamma}_{t}(x)-x}{t}=\frac{\mathrm{e}^{\mathrm{i} t a}-\mathbf{1}}{t} x \mathrm{e}^{-\mathrm{i} t a}+x \frac{\mathrm{e}^{-\mathrm{i} t a}-\mathbf{1}}{t}
$$

for all $t \in \mathbb{R} \backslash\{0\}$. Since $\left(\mathrm{e}^{\mathrm{i} t \xi}-1\right) / t \rightarrow \mathrm{i} \xi$ uniformly on bounded subsets of $\mathbb{R}$ as $t \rightarrow 0$, it follows from Proposition 3.2 that

$$
\frac{\mathrm{e}^{\mathrm{i} t a}-\mathbf{1}}{t} \rightarrow \mathrm{i} a \quad \text { as } t \rightarrow 0
$$

in measure. Similarly, $\mathrm{e}^{-\mathrm{i} t \xi} \rightarrow 1$ uniformly on bounded subsets of $\mathbb{R}$ as $t \rightarrow 0$, and so $\mathrm{e}^{-\mathrm{i} t a} \rightarrow \mathbf{1}$ as $t \rightarrow 0$ in measure. Therefore, since $\tilde{\mathcal{M}}$ is a topological algebra, we find that

$$
\lim _{t \rightarrow 0} \frac{\tilde{\gamma}_{t}(x)-x}{t}=(\mathrm{i} a) x+x(-\mathrm{i} a)=\mathrm{i}[a, x] .
$$

Observe that in the next lemma we do not assume that the space $E$ has order-continuous norm, so the group $\gamma^{E}$ need not be strongly continuous in general. 
Lemma 6.2. Let $E$ be a symmetric BFS on $(0, \infty)$. Given $a \in \tilde{\mathcal{M}}_{h}$, define the group $\gamma^{E}=\left\{\gamma_{t}^{E}\right\}_{t \in \mathbb{R}}$ by $\gamma_{t}^{E}(x)=\mathrm{e}^{\mathrm{i} t a} x \mathrm{e}^{-\mathrm{i} t a}$ for all $x \in E(\mathcal{M}, \tau)$. If $x \in E$ is such that

$$
\lim _{t \rightarrow 0} \frac{\gamma_{t}^{E}(x)-x}{t}
$$

exists with respect to the norm in $E(\mathcal{M}, \tau)$, then $[a, x] \in E(\mathcal{M}, \tau)$ and

$$
\lim _{t \rightarrow 0} \frac{\gamma_{t}^{E}(x)-x}{t}=\mathrm{i}[a, x] .
$$

Proof. Since the embedding of $E(\mathcal{M}, \tau)$ into $\tilde{\mathcal{M}}$ is continuous with respect to the norm topology in $E(\mathcal{M}, \tau)$ and the measure topology in $\tilde{\mathcal{M}}$ (see [15, Lemma 4.4]), this is an immediate consequence of Lemma 6.1.

Corollary 6.3. Suppose that $E$ is a separable r.i. BFS and let $\delta^{E}$ be the infinitesimal generator of the strongly continuous group $\left\{\gamma_{t}^{E}\right\}_{t \in \mathbb{R}}$ in $E(\mathcal{M}, \tau)$ corresponding to $a \in \tilde{\mathcal{M}}_{h}$. If $x \in \operatorname{Dom}\left(\delta^{E}\right)$, then $[a, x] \in E(\mathcal{M}, \tau)$ and $\delta^{E}(x)=\mathrm{i}[a, x]$.

In the proof of Lemma 6.5 we will use following result (see [13, Lemma 5.1]).

Lemma 6.4. Let $E$ be a separable r.i. BFS. If $\left\{q_{n}\right\}_{n=1}^{\infty}$ is a sequence of projections in $\mathcal{M}$ such that $q_{n} \downarrow 0$, then $\left\|q_{n} x\right\|_{E(\mathcal{M}, \tau)} \rightarrow 0$ and $\left\|x q_{n}\right\|_{E(\mathcal{M}, \tau)} \rightarrow 0$ as $n \rightarrow \infty$ for all $x \in E(\mathcal{M}, \tau)$.

Recall that the duality pairing between a non-commutative symmetric space $E(\mathcal{M}, \tau)$ and its Köthe dual space $E(\mathcal{M}, \tau)^{\times}$is given by $\langle x, y\rangle=\tau(x y)$ for all $x \in E(\mathcal{M}, \tau)$ and $y \in E(\mathcal{M}, \tau)^{\times}($see $(2.3))$.

Lemma 6.5. Let $E$ be a separable r.i. BFS. If $a \in \tilde{\mathcal{M}}_{h}, x \in E(\mathcal{M}, \tau)$ and $y \in$ $E(\mathcal{M}, \tau)^{\times}$are such that $[a, x] \in E(\mathcal{M}, \tau)$ and $[a, y] \in E(\mathcal{M}, \tau)^{\times}$, then

$$
\langle[a, x], y\rangle=-\langle x,[a, y]\rangle .
$$

Proof. First we consider the special case that $a \in \mathcal{M}_{h}$. Then $a x, x a \in E(\mathcal{M}, \tau)$ and $a y, y a \in E(\mathcal{M}, \tau)^{\times}$. Hence,

$$
\langle[a, x], y\rangle=\tau(a x y-x a y)=\tau(a x y)-\tau(x a y),
$$

since $a x y, x a y \in L_{1}(\mathcal{M}, \tau)$. Moreover, $\tau(a x y)=\tau(x y a)$, and so

$$
\langle[a, x], y\rangle=\tau(x y a)-\tau(x a y)=\tau(x(y a-a y))=-\langle x,[a, y]\rangle .
$$

Now let $a \in \tilde{\mathcal{M}}_{h}$ be arbitrary. For $n \in \mathbb{N}$, define the spectral projections $p_{n}=e^{a}([-n, n])$ and define $a_{n}=p_{n} a=a p_{n}$. Since $p_{n} \uparrow \mathbf{1}$, it follows from Lemma 6.4 that

$$
\left\|p_{n}[a, x] p_{n}-[a, x]\right\|_{E(\mathcal{M}, \tau)} \rightarrow 0
$$

as $n \rightarrow \infty$. This implies in particular that $\tau\left(p_{n}[a, x] p_{n} y\right) \rightarrow \tau([a, x] y)$ as $n \rightarrow \infty$. On the other hand, for all $n \in \mathbb{N}$, we have $p_{n}[a, x] p_{n}=\left[a_{n}, p_{n} x p_{n}\right]$ and hence, applying the first 
part of the proof to the elements $a_{n} \in \mathcal{M}_{h}, p_{n} x p_{n} \in E(\mathcal{M}, \tau)$ and $y \in E(\mathcal{M}, \tau)^{\times}$, we find that

$$
\begin{aligned}
\tau\left(p_{n}[a, x] p_{n} y\right) & =\tau\left(\left[a_{n}, p_{n} x p_{n}\right] y\right)=-\tau\left(p_{n} x p_{n}\left[a_{n}, y\right]\right) \\
& =-\tau\left(x p_{n}\left[a_{n}, y\right] p_{n}\right)=-\tau\left(x p_{n}[a, y] p_{n}\right) \\
& =-\tau\left(p_{n} x p_{n}[a, y]\right) .
\end{aligned}
$$

By Lemma 6.4 we know that $\left\|p_{n} x p_{n}-x\right\|_{E(\mathcal{M}, \tau)} \rightarrow 0$, and so $\tau\left(p_{n} x p_{n}[a, y]\right) \rightarrow \tau(x[a, y])$ as $n \rightarrow \infty$. Hence, we may conclude that $\tau([a, x] y)=-\tau(x[a, y])$ and the proof is complete.

Next we will use some results from the duality theory of strongly continuous (semi-)groups. For the convenience of the reader we recall the relevant facts (for the details we refer the reader to [19, Chapter XIV]). Suppose that $\{T(t)\}_{t \geqslant 0}$ is a strongly continuous semi-group of linear operators in the Banach space $X$, with infinitesimal generator $A: \operatorname{Dom}(A) \rightarrow X$. The adjoints of the operators $T(t)$ form a semi-group $\left\{T^{*}(t)\right\}_{t \geqslant 0}$ in the dual space $X^{*}$. In general, $\left\{T^{*}(t)\right\}_{t \geqslant 0}$ is not strongly continuous on $X^{*}$. The sundual space $X^{\odot}$ (with respect to $\{T(t)\}_{t \geqslant 0}$ ) is defined by

$$
X^{\odot}=\left\{x^{*} \in X^{*}:\left\|T^{*}(t) x^{*}-x^{*}\right\|_{X^{*}} \rightarrow 0 \text { as } t \downarrow 0\right\},
$$

which is a closed linear subspace of $X^{*}$. Moreover, $X^{\odot}$ separates the points of $X$ and $X^{\odot}=\overline{\operatorname{Dom}\left(A^{*}\right)}$. Defining $T^{\odot}(t)=T^{*}(t)_{\mid X \odot}$ for all $t \geqslant 0$, it is clear that $\left\{T^{\odot}(t)\right\}_{t \geqslant 0}$ is a strongly continuous semi-group in $X^{\odot}$. The generator of $\left\{T^{\odot}(t)\right\}_{t \geqslant 0}$ is denoted by $A^{\odot}$, which is the part of $A^{*}$ in $X^{\odot}$, that is

$$
\operatorname{Dom}\left(A^{\odot}\right)=\left\{x^{*} \in \operatorname{Dom}\left(A^{*}\right): A^{*} x^{*} \in X^{\odot}\right\}
$$

and $A^{\odot} x^{*}=A^{*} x^{*}$ for all $x^{*} \in \operatorname{Dom}\left(A^{\odot}\right)$. Since $\operatorname{Dom}\left(A^{\odot}\right)$ is norm dense in $X^{\odot}$, it follows in particular that $\operatorname{Dom}\left(A^{\odot}\right)$ separates the points of $X$. We shall furthermore need the following observation.

Lemma 6.6. Let $\{T(t)\}_{t \geqslant 0}$ be a strongly continuous semi-group in the Banach space $X$, with generator $A$. If $x, z \in X$ are such that $\left\langle x, A^{\odot} y\right\rangle=\langle z, y\rangle$ for all $y \in \operatorname{Dom}\left(A^{\odot}\right)$, then $x \in \operatorname{Dom}(A)$ and $A x=z$.

Proof. Fix $y \in \operatorname{Dom}\left(A^{\odot}\right)$ and define $F(t)=\langle T(t) x, y\rangle$ for $t \geqslant 0$. For all $0 \leqslant t \in \mathbb{R}$ and $h>0$ we have

$$
\begin{aligned}
\frac{F(t+h)-F(t)}{h} & =\frac{1}{h}\langle T(t+h) x-T(t) x, y\rangle \\
& =\left\langle x, \frac{T^{*}(t+h) y-T^{*}(t) y}{h}\right\rangle .
\end{aligned}
$$

Since $T^{*}(t) y=T^{\odot}(t) y \in \operatorname{Dom}\left(A^{\odot}\right)$, it follows from the assumption on $x$ and $z$ that

$$
\lim _{h \downarrow 0} \frac{F(t+h)-F(t)}{h}=\left\langle x, A^{\odot} T^{*}(t) y\right\rangle=\left\langle z, T^{*}(t) y\right\rangle=\langle T(t) z, y\rangle .
$$


The function $t \mapsto T(t) z$ is continuous and $F(0)=\langle x, y\rangle$, so we may conclude that

$$
F(t)=\langle x, y\rangle+\int_{0}^{t}\langle T(s) z, y\rangle \mathrm{d} s=\left\langle x+\int_{0}^{t} T(s) z \mathrm{~d} s, y\right\rangle
$$

for all $t \geqslant 0$. Since this holds for all $y \in \operatorname{Dom}\left(A^{\odot}\right)$ and $\operatorname{Dom}\left(A^{\odot}\right)$ separates the points of $X$, it follows that

$$
T(t) x=x+\int_{0}^{t} T(s) z \mathrm{~d} s
$$

for all $t \geqslant 0$. Using again the fact that $s \mapsto T(s) z$ is continuous, this implies that

$$
\lim _{t \downarrow 0} \frac{T(t) x-x}{t}=z .
$$

Hence, $x \in \operatorname{Dom}(A)$ and $A x=z$.

Now we return to the situation where $E$ is a separable r.i. BFS and where the $\mathbb{R}$-flow $\gamma=\left\{\gamma_{t}\right\}_{t \in \mathbb{R}}$ on $(\mathcal{M}, \tau)$ is given by (6.1) for some $a \in \tilde{\mathcal{M}}_{h}$. As observed in (4.2), we have $\left(\gamma_{t}^{E}\right)^{*}=\gamma_{-t}^{E^{\times}}$for all $t \in \mathbb{R}$.

Lemma 6.7. If $y \in \operatorname{Dom}\left(\left(\delta^{E}\right) \odot\right)$, then $[a, y] \in E(\mathcal{M}, \tau)^{\times}$and $\left(\delta^{E}\right)^{\odot}(y)=-\mathrm{i}[a, y]$.

Proof. Let $y \in \operatorname{Dom}\left(\left(\delta^{E}\right)^{\odot}\right)$ be given. By definition we have

$$
\left(\delta^{E}\right)^{\odot} y=\lim _{t \rightarrow 0} \frac{\left(\gamma_{t}^{E}\right)^{*}(y)-y}{y}=\lim _{t \rightarrow 0} \frac{\gamma_{-t}^{E^{\times}}(y)-y}{t} .
$$

An application of Lemma 6.2 to the symmetric operator space $E(\mathcal{M}, \tau)^{\times}$and the group $\left\{\gamma_{-t}^{E^{\times}}\right\}_{t \in \mathbb{R}}$ yields $[-a, y] \in E(\mathcal{M}, \tau)^{\times}$and $\left(\delta^{E}\right)^{\odot}(y)=-\mathrm{i}[a, y]$.

Now we are in a position to prove the main result in this section.

Theorem 6.8. Let $E$ be a separable r.i. BFS on $(0, \infty)$. Suppose that $a \in \tilde{\mathcal{M}}_{h}$ and let $\gamma^{E}=\left\{\gamma_{t}^{E}\right\}_{t \in \mathbb{R}}$ be the strongly continuous group in $E(\mathcal{M}, \tau)$ defined by $\gamma_{t}(x)=\mathrm{e}^{\mathrm{i} t a} x \mathrm{e}^{-\mathrm{i} t a}$ for all $x \in E(\mathcal{M}, \tau)$. Then the infinitesimal generator $\delta^{E}$ of $\gamma^{E}$ is given by

$$
\operatorname{Dom}\left(\delta^{E}\right)=\{x \in E(\mathcal{M}, \tau):[a, x] \in E(\mathcal{M}, \tau)\}
$$

and $\delta^{E}(x)=\mathrm{i}[a, x]$ for all $x \in \operatorname{Dom}\left(\delta^{E}\right)$.

Proof. From Corollary 6.3 we know that $\operatorname{Dom}\left(\delta^{E}\right)$ is contained in $\{x \in E(\mathcal{M}, \tau)$ : $[a, x] \in E(\mathcal{M}, \tau)\}$ and that $\delta^{E}(x)=\mathrm{i}[a, x]$ for all $x \in \operatorname{Dom}\left(\delta^{E}\right)$. Now assume that $x \in E(\mathcal{M}, \tau)$ is such that $[a, x] \in E(\mathcal{M}, \tau)$. Given $y \in \operatorname{Dom}\left(\left(\delta^{E}\right)^{\odot}\right)$, it follows from Lemma 6.7 that $\left(\delta^{E}\right)^{\odot}(y)=-\mathrm{i}[a, y]$. Using the result of Lemma 6.5 we find that

$$
\left\langle x,\left(\delta^{E}\right)^{\odot}(y)\right\rangle=-\mathrm{i}\langle x,[a, y]\rangle=\mathrm{i}\langle[a, x], y\rangle .
$$

Since this holds for all $y \in \operatorname{Dom}\left(\left(\delta^{E}\right)^{\odot}\right)$, it follows from Lemma 6.6 that $x \in \operatorname{Dom}\left(\delta^{E}\right)$ and $\delta^{E}(x)=\mathrm{i}[a, x]$. The proof is complete. 
The next corollary follows from a combination of Theorems 5.7 and 6.8.

Corollary 6.9. Suppose that $E$ is a separable r.i. BFS and that $a \in \tilde{\mathcal{M}}_{h}$. If $x^{*}=$ $x \in E(\mathcal{M}, \tau)$ such that $[a, x] \in E(\mathcal{M}, \tau)$, then $[a, f(x)] \in E(\mathcal{M}, \tau)$ for all $f \in \Psi^{-1}\left(\mathfrak{C}_{0}\right)$ and there exists a constant $C_{f}>0$, only depending on the function $f$, such that $\|[a, f(x)]\|_{E(\mathcal{M}, \tau)} \leqslant C_{f}\|[a, x]\|_{E(\mathcal{M}, \tau)}$.

Proof. Replacing the function $f(\lambda)$ by $f(\lambda)-f(0)$, we may assume, without loss of generality, that $f(0)=0$. Then the result of Theorem 5.7 is applicable to the $\mathbb{R}$-flow $\gamma^{E}$ given by (6.1). Suppose that $x^{*}=x \in E(\mathcal{M}, \tau)$ with $[a, x] \in E(\mathcal{M}, \tau)$. By Theorem 6.8, this implies that $x \in \operatorname{Dom}\left(\delta^{E}\right)$ and from Theorem 5.7 it follows that $f(x) \in \operatorname{Dom}\left(\delta^{E}\right)$. Using Theorem 6.8 once more, it follows that $[a, f(x)] \in E(\mathcal{M}, \tau)$. Moreover, (5.9) and Proposition 5.3 (ii) imply that

$$
\begin{aligned}
\|[a, f(x)]\|_{E(\mathcal{M}, \tau)} & =\left\|\delta^{E}(f(x))\right\|_{E(\mathcal{M}, \tau)}=\left\|T_{\psi_{f}, x}^{x, x}\left(\delta^{E}(x)\right)\right\|_{E(\mathcal{M}, \tau)} \\
& \leqslant\left\|T_{\psi_{f}}^{x, x}\right\|_{\mathcal{L}(E(\mathcal{M}, \tau))}\left\|\delta^{E}(x)\right\|_{E(\mathcal{M}, \tau)} \\
& \leqslant\left\|\psi_{f}\right\|_{\mathfrak{A}_{0}}\|[a, x]\|_{E(\mathcal{M}, \tau)}
\end{aligned}
$$

so we may take $C_{f}=\left\|\psi_{f}\right\|_{\mathfrak{A}_{0}}$.

\section{7. $\mathbb{R}$-flows induced by self-adjoint operators}

In this section we shall assume that $D: \operatorname{Dom}(D) \rightarrow \mathcal{H}$ is a self-adjoint operator. We shall furthermore assume that

(a) $\mathrm{e}^{\mathrm{i} t D} x \mathrm{e}^{-\mathrm{i} t D} \in \mathcal{M}$ for all $x \in \mathcal{M}$ and all $t \in \mathbb{R}$,

(b) $\tau\left(\mathrm{e}^{\mathrm{i} t D} x \mathrm{e}^{-\mathrm{i} t D}\right)=\tau(x)$ for all $0 \leqslant x \in \mathcal{M}$ and all $t \in \mathbb{R}$.

Note that any self-adjoint operator $D$ that is affiliated with $\mathcal{M}$ automatically satisfies these two conditions. Defining

$$
\gamma_{t}(x)=\mathrm{e}^{\mathrm{i} t D} x \mathrm{e}^{-\mathrm{i} t D}, \quad x \in \mathcal{M}, \quad t \in \mathbb{R}
$$

the argument used at the beginning of $\S 6$ shows that $\gamma=\left\{\gamma_{t}\right\}_{t \in \mathbb{R}}$ is an $\mathbb{R}$-flow in $(\mathcal{M}, \tau)$. For any separable r.i. BFS $E$ on $(0, \infty)$ we consider the corresponding strongly continuous group $\gamma^{E}=\left\{\gamma_{t}^{E}\right\}_{t \in \mathbb{R}}$ of isometries in $E(\mathcal{M}, \tau)$ (see Corollary 4.1), with infinitesimal generator $\delta^{E}$. In this general setting, it is harder to obtain a description of $\operatorname{Dom}\left(\delta^{E}\right)$ than in the previous section (where $D$ is assumed to be $\tau$-measurable). However, it turns out to be possible to give an analogous description of a core of $\delta^{E}$.

Example 7.1. A simple but important illustration of the situation described above is the following classical commutative example. Let $\mathcal{H}=L_{2}(\mathbb{R})$, with respect to Lebesgue measure in $\mathbb{R}$, and $D$ be the operator $\mathrm{d}\left(\mathrm{i}^{-1}\right) / \mathrm{d} t$ in $\mathcal{H}$ with domain the Sobolev space $W^{1,2}(\mathbb{R})$. The unitary group $\left\{\mathrm{e}^{\mathrm{i} t D}\right\}_{t \in \mathbb{R}}$ in $\mathcal{H}$ is the translation group given by 
$\mathrm{e}^{\mathrm{i} t D} g(s)=g(s+t)$ for all $g \in \mathcal{H}$ and $s, t \in \mathbb{R}$. Let $\mathcal{M}=L_{\infty}(\mathbb{R})$, acting on $\mathcal{H}$ via multiplication. Defining the trace $\tau$ by

$$
\tau(f)=\int_{\mathbb{R}} f(s) \mathrm{d} s \quad \text { for all } 0 \leqslant f \in \mathcal{M},
$$

it is well known that $(\mathcal{M}, \tau)$ is a semi-finite von Neumann algebra on $\mathcal{H}$. The operator $D$ is not affiliated with $\mathcal{M}$. A simple computation shows that $\left(\mathrm{e}^{\mathrm{i} t D} f \mathrm{e}^{-\mathrm{i} t D}\right)(s)=f(s+t)$ for all $f \in \mathcal{M}=L_{\infty}(\mathbb{R})$ and $s, t \in \mathbb{R}$. Hence, conditions (a) and (b) are both satisfied and the corresponding $\mathbb{R}$-flow $\left\{\gamma_{t}\right\}_{t \in \mathbb{R}}$ in $\mathcal{M}$ is the translation group in $L_{\infty}(\mathbb{R})$.

In the proof of the main result of this section it will be convenient to have the following technical observation available. Suppose that $f:[0,1] \rightarrow \mathcal{M}$ is a strongly continuous function, that is, for each $\xi \in \mathcal{H}$ the function $t \mapsto f(t) \xi$ is continuous from $[0,1]$ into $\mathcal{H}$. The range of $f$ is norm bounded in $\mathcal{M}$. For each $\xi \in \mathcal{H}$, the $(\mathcal{H}$-valued) Riemann integral

$$
\int_{0}^{1} f(t) \xi \mathrm{d} t
$$

is a well-defined element of $\mathcal{H}$. The linear and bounded map

$$
\xi \mapsto \int_{0}^{1} f(t) \xi \mathrm{d} t
$$

is denoted by

$$
(s o)-\int_{0}^{1} f(t) \mathrm{d} t
$$

Since $\mathcal{M}$ is strongly closed, it is evident that

$$
(s o)-\int_{0}^{1} f(t) \mathrm{d} t \in \mathcal{M} .
$$

If $E$ is a symmetric BFS and if $f:[0,1] \rightarrow E(\mathcal{M}, \tau)$ is a function which is continuous with respect to the norm in $E(\mathcal{M}, \tau)$, then the $E(\mathcal{M}, \tau)$-valued Riemann integral

$$
(E)-\int_{0}^{1} f(t) \mathrm{d} t
$$

exists in $E(\mathcal{M}, \tau)$. In this situation the following lemma holds.

Lemma 7.2. If $f:[0,1] \rightarrow E(\mathcal{M}, \tau) \cap \mathcal{M}$ is a strongly continuous function which is also continuous with respect to the norm in $E(\mathcal{M}, \tau)$, then

$$
(s o)-\int_{0}^{1} f(t) \mathrm{d} t=(E)-\int_{0}^{1} f(t) \mathrm{d} t .
$$

Proof. Put

$$
x=(s o)-\int_{0}^{1} f(t) \mathrm{d} t \quad \text { and } \quad y=(E)-\int_{0}^{1} f(t) \mathrm{d} t .
$$


For $n=1,2, \ldots$ we define the Riemann sums

$$
z_{n}=\frac{1}{n} \sum_{k=1}^{n} f\left(\frac{k}{n}\right)
$$

By the definition of the integrals we have $z_{n} \rightarrow x$ strongly and $\left\|z_{n}-y\right\|_{E(\mathcal{M}, \tau)} \rightarrow 0$ as $n \rightarrow \infty$. Since $\left\|z_{n}\right\|_{\infty} \leqslant \max _{0 \leqslant t \leqslant 1}\|f(t)\|_{\infty}$, and since the strong topology coincides with the ultra-strong topology on norm-bounded subsets of $\mathcal{L}(\mathcal{H})$, it follows that $z_{n} \rightarrow x$ ultra-strongly, and so ultra-weakly, as $n \rightarrow \infty$. Hence, $z_{n} \rightarrow x$ with respect to $\sigma\left(\mathcal{M}, L_{1}\right)$. Furthermore, norm convergence of $\left\{z_{n}\right\}_{n=1}^{\infty}$ to $y$ in $E(\mathcal{M}, \tau)$ implies that $z_{n} \rightarrow y$ as $n \rightarrow \infty$ with respect to $\sigma\left(E, E^{\times}\right)$. Consequently, $z_{n} \rightarrow x$ and $z_{n} \rightarrow y$ with respect to $\sigma\left(L_{1}+L_{\infty}, L_{1} \cap L_{\infty}\right)$ as $n \rightarrow \infty$. Since $L_{1}(\mathcal{M}, \tau) \cap \mathcal{M}$ separates the points of $L_{1}(\mathcal{M}, \tau)+$ $\mathcal{M}$, we may conclude that $x=y$.

Given the separable r.i. BFS $E$, let $\gamma^{E}$ be the strongly continuous group in $E(\mathcal{M}, \tau)$ induced by the $\mathbb{R}$-flow given by (7.1). If $x \in \mathcal{L}(\mathcal{H})$ with the property that $x(\operatorname{Dom}(D)) \subseteq$ $\operatorname{Dom}(D)$, then we define

$$
[D, x](\xi)=D x(\xi)-x D(\xi), \quad \xi \in \operatorname{Dom}(D) .
$$

If the operator $[D, x]$ is closable, then we denote its closure, with some abuse of notation, also by $[D, x]$. In particular, a statement like $[D, x] \in \mathcal{M}$ has to be interpreted as saying that the operator $[D, x]$, defined by $(7.2)$, is closable and its closure belongs to $\mathcal{M}$. Recall that the subspace $F$, defined in (4.5), is given by

$$
F=\left\{x \in \operatorname{Dom}\left(\delta^{E}\right) \cap \mathcal{M}: \delta^{E}(x) \in \mathcal{M}\right\}
$$

and is a core of the generator $\delta^{E}$ (see Theorem 4.3). In this situation the following theorem holds.

Theorem 7.3. The subspace $F$ of $E(\mathcal{M}, \tau) \cap \mathcal{M}$, defined by (4.5), is also given by

$$
F=\{x \in E(\mathcal{M}, \tau) \cap \mathcal{M}: x(\operatorname{Dom}(D)) \subseteq \operatorname{Dom}(D),[D, x] \in E(\mathcal{M}, \tau) \cap \mathcal{M}\}
$$

and $\delta^{E}(x)=\mathrm{i}[D, x]$ for all $x \in F$.

Proof. First suppose that $x \in E(\mathcal{M}, \tau) \cap \mathcal{M}$ satisfies $x(\operatorname{Dom}(D)) \subseteq \operatorname{Dom}(D)$ and $[D, x] \in E(\mathcal{M}, \tau) \cap \mathcal{M}$. Take $\xi \in \operatorname{Dom}(D)$ and define $u(t)=\gamma_{t}^{E}(x) \xi$ for all $t \in \mathbb{R}$. A simple computation shows that

$$
\frac{u(t+h)-u(t)}{h}=\mathrm{e}^{\mathrm{i}(t+h) D} x \frac{\mathrm{e}^{-\mathrm{i} h D}\left(\mathrm{e}^{-\mathrm{i} t D} \xi\right)-\mathrm{e}^{-\mathrm{i} t D} \xi}{h}+\mathrm{e}^{\mathrm{i} t D} \frac{\mathrm{e}^{\mathrm{i} h D} x \mathrm{e}^{-\mathrm{i} t D} \xi-x \mathrm{e}^{-\mathrm{i} t D} \xi}{h}
$$

for all $h \neq 0$ and $t \in \mathbb{R}$. Since $\xi \in \operatorname{Dom}(D)$ implies that $\mathrm{e}^{-\mathrm{i} t D} \xi \in \operatorname{Dom}(D)$ and $x \mathrm{e}^{-\mathrm{i} t D} \xi \in$ $\operatorname{Dom}(D)$, we find that

$$
\begin{aligned}
u^{\prime}(t) & =\lim _{h \rightarrow 0} \frac{u(t+h)-u(t)}{h} \\
& =\mathrm{e}^{\mathrm{i} t D} x\left(-\mathrm{i} D \mathrm{e}^{-\mathrm{i} t D} \xi\right)+\mathrm{e}^{\mathrm{i} t D}(\mathrm{i} D)\left(x \mathrm{e}^{-\mathrm{i} t D} \xi\right) \\
& =\mathrm{ie}^{\mathrm{i} t D}[D, x] \mathrm{e}^{-\mathrm{i} t D} \xi
\end{aligned}
$$


for all $t \in \mathbb{R}$. Since $[D, x] \in \mathcal{L}(\mathcal{H})$, the function $t \mapsto \mathrm{ie}^{\mathrm{i} t D}[D, x] \mathrm{e}^{-\mathrm{i} t D} \xi$ is continuous from $\mathbb{R}$ into $\mathcal{H}$, the fundamental theorem of calculus implies that

$$
\gamma_{t}^{E}(x) \xi-x(\xi)=u(t)-u(0)=\mathrm{i} \int_{0}^{t} \mathrm{e}^{\mathrm{i} s D}[D, x] \mathrm{e}^{-\mathrm{i} s D} \xi \mathrm{d} s
$$

for all $t \in \mathbb{R}$ and all $\xi \in \operatorname{Dom}(D)$. Since $[D, x] \in \mathcal{L}(\mathcal{H})$, the function $t \mapsto \mathrm{ie}^{\mathrm{i} t D}[D, x] \mathrm{e}^{-\mathrm{i} t D}$ is strongly continuous, and so

$$
(s o)-\mathrm{i} \int_{0}^{t} \mathrm{e}^{\mathrm{i} s D}[D, x] \mathrm{e}^{-\mathrm{i} s D} \mathrm{~d} s \in \mathcal{L}(\mathcal{H})
$$

and it follows from (7.3) that this operator coincides with $\gamma_{t}^{E}(x)-x$ on the dense set $\operatorname{Dom}(D)$. Hence,

$$
\gamma_{t}^{E}(x)-x=(s o)-\mathrm{i} \int_{0}^{t} \mathrm{e}^{\mathrm{i} s D}[D, x] \mathrm{e}^{-\mathrm{i} s D} \mathrm{~d} s .
$$

Furthermore, since $[D, x] \in E(\mathcal{M}, \tau)$, the function $t \mapsto \mathrm{ie}^{\mathrm{i} t D}[D, x] \mathrm{e}^{-\mathrm{i} t D}$ is continuous with respect to the norm in $E(\mathcal{M}, \tau)$ (see Corollary 4.1) and so, by Lemma 7.2, we may conclude that

$$
(s o)-\mathrm{i} \int_{0}^{t} \mathrm{e}^{\mathrm{i} s D}[D, x] \mathrm{e}^{-\mathrm{i} s D} \mathrm{~d} s=(E)-\mathrm{i} \int_{0}^{t} \mathrm{e}^{\mathrm{i} s D}[D, x] \mathrm{e}^{-\mathrm{i} s D} \mathrm{~d} s .
$$

Hence,

$$
\gamma_{t}^{E}(x)-x=(E)-\mathrm{i} \int_{0}^{t} \mathrm{e}^{\mathrm{i} s D}[D, x] \mathrm{e}^{-\mathrm{i} s D} \mathrm{~d} s .
$$

From this equality it follows immediately that $x \in \operatorname{Dom}\left(\delta^{E}\right)$ and that $\delta^{E}(x)=\mathrm{i}[D, x] \in$ $\mathcal{M}$. In particular, $x \in F$.

For the proof of the converse inclusion, assume that $x \in \operatorname{Dom}\left(\delta^{E}\right) \cap \mathcal{M}$ and $\delta^{E}(x) \in \mathcal{M}$. Then we know that

$$
\gamma_{t}^{E}(x)-x=(E)-\mathrm{i} \int_{0}^{t} \gamma_{s}^{E}\left(\delta^{E}(x)\right) \mathrm{d} s
$$

for all $t \in \mathbb{R}$. Since $\delta^{E}(x) \in \mathcal{L}(\mathcal{H})$, the function $t \mapsto \gamma_{s}^{E}\left(\delta^{E}(x)\right)=\mathrm{e}^{\mathrm{i} t D} \delta^{E}(x) \mathrm{e}^{-t D}$ is strongly continuous, and so it follows from Lemma 7.2 that

$$
(E)-\mathrm{i} \int_{0}^{t} \gamma_{s}^{E}\left(\delta^{E}(x)\right) \mathrm{d} s=(s o)-\mathrm{i} \int_{0}^{t} \gamma_{s}^{E}\left(\delta^{E}(x)\right) \mathrm{d} s .
$$

Hence,

$$
\gamma_{t}^{E}(x) \xi-x \xi=\mathrm{i} \int_{0}^{t} \mathrm{e}^{\mathrm{i} t D} \delta^{E}(x) \mathrm{e}^{-t D} \xi \mathrm{d} s
$$

for all $\xi \in \mathcal{H}$. This implies that

$$
\lim _{t \rightarrow 0} \frac{\gamma_{t}^{E}(x) \xi-x \xi}{t}=\delta^{E}(x) \xi
$$


for all $\xi \in \mathcal{H}$ (where the limit is taken with respect to the norm of $\mathcal{H}$ ). Take $\xi \in \operatorname{Dom}(D)$. Writing

$$
\frac{\mathrm{e}^{\mathrm{i} t D} x \xi-x \xi}{t}=\frac{\mathrm{e}^{\mathrm{i} t D} x \mathrm{e}^{-t D} \xi-x \xi}{t}-\mathrm{e}^{\mathrm{i} t D} x \frac{\mathrm{e}^{-\mathrm{i} t D} \xi-\xi}{t},
$$

we see that

$$
\lim _{t \rightarrow 0} \frac{\mathrm{e}^{\mathrm{i} t D} x \xi-x \xi}{t}=\delta^{E}(x) \xi+\mathrm{i} x D \xi .
$$

Hence, $x \xi \in \operatorname{Dom}(D)$ and $\mathrm{i} D(x \xi)=\delta^{E}(x) \xi+\mathrm{i} x D \xi$. This also shows that $\mathrm{i}[D, x] \xi=$ $\delta^{E}(x) \xi$ for all $\xi \in \operatorname{Dom}(D)$, and so $[D, x]$ is closable and its closure is given by $-\mathrm{i} \delta^{E}(x) \in E(\mathcal{M}, \tau) \cap \mathcal{M}$, that is, $[D, x] \in E(\mathcal{M}, \tau) \cap \mathcal{M}$. The proof is complete.

Remark 7.4. The same situation as in Theorem 7.3. If $x \in \operatorname{Dom}\left(\delta^{E}\right) \cap \mathcal{M}$, then in general the commutator $[D, x]$ cannot be defined via (7.2). To see this we use the commutative situation explained in Example 7.1. For the BFS $E$ we take $L_{1}(0, \infty)$. The space $L_{1}(\mathcal{M}, \tau)$ may be identified with $L_{1}(\mathbb{R})$, acting on $\mathcal{H}=L_{2}(\mathbb{R})$ as (unbounded) multiplication operators. The group $\gamma^{E}$ is the translation group in $L_{1}(\mathbb{R})$. The infinitesimal generator $\delta^{E}$ of this group has as domain the Sobolev space $W^{1,1}(\mathbb{R})$ and is given by $\delta^{E}(f)=f^{\prime}$, the weak derivative of $f$. As is well known, $W^{1,1}(\mathbb{R}) \subseteq C_{0}(\mathbb{R})$, where $C_{0}(\mathbb{R})$ is the space of all continuous complex-valued functions on $\mathbb{R}$ vanishing at infinity (for elementary properties of Sobolev spaces we refer the reader to, for example, $[\mathbf{9}])$. Hence, $\operatorname{Dom}\left(\delta^{E}\right) \cap \mathcal{M}=\operatorname{Dom}\left(\delta^{E}\right)=W^{1,1}(\mathbb{R})$. Take $f \in W^{1,1}(\mathbb{R})$ and $g \in \operatorname{Dom}(D)=W^{1,2}(\mathbb{R})$. Since $f$ is bounded, it is evident that $f g \in L_{2}(\mathbb{R})$. Moreover, $(f g)^{\prime}=f^{\prime} g+f g^{\prime}$. Since $g^{\prime} \in L_{2}(\mathbb{R})$ and $f$ is bounded, it is also clear that $f g^{\prime} \in L_{2}(\mathbb{R})$. Consequently, the condition that $f g \in \operatorname{Dom}(D)$ is equivalent to $f^{\prime} g \in L_{2}(\mathbb{R})$. However, it is not difficult to construct a function $f \in W^{1,1}(\mathbb{R})$ with the property that $f^{\prime} g \notin L_{2}(\mathbb{R})$ for all $0 \neq g \in W^{1,2}(\mathbb{R})$.

This example shows that it is possible that $x \in \operatorname{Dom}\left(\delta^{E}\right) \cap \mathcal{M}$ is such that $x \xi \in$ $\operatorname{Dom}(D)$, with $\xi \in \operatorname{Dom}(D)$, only holds for $\xi=0$. Therefore, it is essential to include the condition that $\delta^{E}(x) \in \mathcal{M}$ in the definition (4.5) of the set $F$.

Using the result of the above theorem in combination with Theorem 5.7, we are able to obtain certain commutator estimates, similar to the results obtained in [7] in the setting of symmetrically normed ideals of compact operators (that is, for the case that $\mathcal{M}$ is a type-I factor). In the formulation of the next result we use the function space $\Psi^{-1}\left(\mathfrak{C}_{0}\right)$, as introduced before Proposition 5.6.

Corollary 7.5. Let $E$ be a separable r.i. BFS and suppose that $D$ is a self-adjoint operator in $\mathcal{H}$, satisfying conditions (a) and (b) at the beginning of this section. If $x^{*}=x \in E(\mathcal{M}, \tau) \cap \mathcal{M}$ is such that $[D, x] \in E(\mathcal{M}, \tau) \cap \mathcal{M}$, then $[D, f(x)] \in E(\mathcal{M}, \tau) \cap \mathcal{M}$ for all $f \in \Psi^{-1}\left(\mathfrak{C}_{0}\right)$ and there exists a constant $C_{f}>0$, depending only on the function $f$, such that

$$
\begin{aligned}
\|[D, f(x)]\|_{E(\mathcal{M}, \tau) \cap \mathcal{M}} & \leqslant C_{f}\|[D, x]\|_{E(\mathcal{M}, \tau) \cap \mathcal{M}}, \\
\|[D, f(x)]\|_{E(\mathcal{M}, \tau)}(\mathcal{M}, \tau) & \leqslant C_{f}\|[D, x]\|_{E(\mathcal{M}, \tau)} .
\end{aligned}
$$


Proof. As in the proof of Corollary 6.9, we may assume, without loss of generality, that $f(0)=0$. Given $x^{*}=x \in E(\mathcal{M}, \tau) \cap \mathcal{M}$ with $[D, x] \in E(\mathcal{M}, \tau) \cap \mathcal{M}$, it follows from Theorem 7.3 that $x \in \operatorname{Dom}\left(\delta^{E}\right) \cap \mathcal{M}$ and $[D, x]=\delta^{E}(x) \in \mathcal{M}$. Using Theorem 5.7, this implies that $f(x) \in \operatorname{Dom}\left(\delta^{E}\right)$ and $\delta^{E}(f(x))=T_{\psi_{f}}^{x, x}\left(\delta^{E}(x)\right)$. Since $x \in \mathcal{M}$ and $f$ is bounded on bounded subsets of $\mathbb{R}$, it is clear that $f(x) \in \mathcal{M}$. Furthermore, it follows from Lemma 5.5 that $T_{\psi_{f}}^{x, x}\left(\delta^{E}(x)\right) \in E(\mathcal{M}, \tau) \cap \mathcal{M}$ and

$$
\left\|T_{\psi_{f}}^{x, x}\left(\delta^{E}(x)\right)\right\|_{E(\mathcal{M}, \tau) \cap \mathcal{M}} \leqslant\left\|\psi_{f}\right\|_{\mathfrak{A}_{0}}\left\|\delta^{E}(x)\right\|_{E(\mathcal{M}, \tau) \cap \mathcal{M}} .
$$

Applying Theorem 7.3 once more, we may conclude that $f(x)(\operatorname{Dom}(D)) \subseteq \operatorname{Dom}(D)$, that $[D, f(x)]=\delta^{E}(f(x)) \in E(\mathcal{M}, \tau) \cap \mathcal{M}$ and that

$$
\|[D, f(x)]\|_{E(\mathcal{M}, \tau) \cap \mathcal{M}} \leqslant\left\|\psi_{f}\right\|_{\mathfrak{A}_{0}}\|[D, x]\|_{E(\mathcal{M}, \tau) \cap \mathcal{M}} .
$$

Moreover, from Proposition 5.3 (ii) we know that $\left\|T_{\psi_{f}}^{x, x}\right\|_{\mathcal{L}(E(\mathcal{M}, \tau))} \leqslant\left\|\psi_{f}\right\|_{\mathfrak{A}_{0}}$, from which it follows that $\|[D, f(x)]\|_{E(\mathcal{M}, \tau)} \leqslant C_{f}\|[D, x]\|_{E(\mathcal{M}, \tau)}$.

We end the paper with an interesting application of Corollary 7.5. We assume that $A$ and $B$ are two self-adjoint operators in $\mathcal{H}$ satisfying the following two conditions:

(i) for all $x \in \mathcal{M}$ and $t \in \mathbb{R}$ we have $\mathrm{e}^{\mathrm{i} t A} x \mathrm{e}^{-\mathrm{i} t A}, \mathrm{e}^{\mathrm{i} t B} x \mathrm{e}^{-\mathrm{i} t B}, \mathrm{e}^{\mathrm{i} t A} x \mathrm{e}^{\mathrm{i} t B} \in \mathcal{M}$;

(ii) $\tau\left(\mathrm{e}^{\mathrm{i} t A} x \mathrm{e}^{-\mathrm{i} t A}\right)=\tau\left(\mathrm{e}^{\mathrm{i} t B} x \mathrm{e}^{-\mathrm{i} t B}\right)=\tau(x)$ for all $0 \leqslant x \in \mathcal{M}$ and all $t \in \mathbb{R}$.

Furthermore, we assume that $E$ is a separable r.i. BFS. If $x \in \mathcal{L}(\mathcal{H})$ satisfies $x(\operatorname{Dom}(B)) \subseteq \operatorname{Dom}(A)$, then the operator $A x+x B$ is well defined on $\operatorname{Dom}(B)$. If this operator is closable, then we denote its closure also by $A x+x B$. Under these conditions the following result holds.

Proposition 7.6. Suppose that $f \in \Psi^{-1}\left(\mathfrak{C}_{0}\right)$ is odd. If $x \in E(\mathcal{M}, \tau) \cap \mathcal{M}$ is such that $x(\operatorname{Dom}(B)) \subseteq \operatorname{Dom}(A)$ and $A x+x B \in E(\mathcal{M}, \tau) \cap \mathcal{M}$, then $f(x)(\operatorname{Dom}(B)) \subseteq \operatorname{Dom}(A)$ and $A f(x)+f(x) B \in E(\mathcal{M}, \tau) \cap \mathcal{M}$. Moreover, there exists a constant $C_{f}>0$, depending only on $f$, such that

$$
\left.\begin{array}{c}
\|A f(x)+f(x) B\|_{E(\mathcal{M}, \tau) \cap \mathcal{M}} \leqslant C_{f}\|A x+x B\|_{E(\mathcal{M}, \tau) \cap \mathcal{M} ;} \\
\|A f(x)+f(x) B\|_{E(\mathcal{M}, \tau)} \leqslant C_{f}\|A x+x B\|_{E(\mathcal{M}, \tau) .}
\end{array}\right\}
$$

Proof. On the Hilbert space $\mathcal{H} \oplus \mathcal{H}$ we consider the semi-finite von Neumann algebra $\mathcal{M}_{2}=\mathcal{M} \otimes M_{2}(\mathbb{C})=M_{2}(\mathcal{M})$, equipped with the trace $\tau_{2}$ given by

$$
\tau_{2}\left(\left[x_{j k}\right]\right)=\tau\left(x_{11}\right)+\tau\left(x_{22}\right), 0 \leqslant\left[x_{j k}\right] \in \mathcal{M}_{2} .
$$

Define the self-adjoint operator $D$ in $\mathcal{H} \oplus \mathcal{H}$ by $\operatorname{Dom}(D)=\operatorname{Dom}(A) \oplus \operatorname{Dom}(B)$ and $D(\xi, \eta)=(A \xi,-B \eta)$ for all $(\xi, \eta) \in \operatorname{Dom}(D)$. Note that $\mathrm{e}^{\mathrm{i} t D}(\xi, \eta)=\left(\mathrm{e}^{\mathrm{i} t A} \xi, \mathrm{e}^{-\mathrm{i} t B} \eta\right)$ for all $(\xi, \eta) \in \mathcal{H} \oplus \mathcal{H}$. Consequently, if $\left[x_{j k}\right] \in \mathcal{M}_{2}$, then

$$
\mathrm{e}^{\mathrm{i} t D}\left[\begin{array}{ll}
x_{11} & x_{12} \\
x_{21} & x_{22}
\end{array}\right] \mathrm{e}^{-\mathrm{i} t D}=\left[\begin{array}{cc}
\mathrm{e}^{\mathrm{i} t A} x_{11} \mathrm{e}^{-\mathrm{i} t A} & \mathrm{e}^{\mathrm{i} t A} x_{12} \mathrm{e}^{\mathrm{i} t B} \\
\mathrm{e}^{-\mathrm{i} t B} x_{21} \mathrm{e}^{-\mathrm{i} t A} & \mathrm{e}^{-\mathrm{i} t B} x_{22} \mathrm{e}^{\mathrm{i} t B}
\end{array}\right] .
$$


Conditions (i) and (ii) above imply that

(i) $\mathrm{e}^{\mathrm{i} t D}\left[x_{j k}\right] \mathrm{e}^{-\mathrm{i} t D} \in \mathcal{M}_{2}$ for all $\left[x_{j k}\right] \in \mathcal{M}_{2}$ and all $t \in \mathbb{R}$,

(ii) if $0 \leqslant\left[x_{j k}\right] \in \mathcal{M}_{2}$, then

$$
\begin{aligned}
\tau_{2}\left(\mathrm{e}^{\mathrm{i} t D}\left[x_{j k}\right] \mathrm{e}^{-\mathrm{i} t D}\right) & =\tau\left(\mathrm{e}^{\mathrm{i} t A} x_{11} \mathrm{e}^{-\mathrm{i} t A}\right)+\tau\left(\mathrm{e}^{-\mathrm{i} t B} x_{22} \mathrm{e}^{\mathrm{i} t B}\right) \\
& =\tau\left(x_{11}\right)+\tau\left(x_{22}\right) \\
& =\tau_{2}\left(\left[x_{j k}\right]\right) .
\end{aligned}
$$

Hence, conditions (a) and (b) at the beginning of this section are satisfied. Now suppose that $x \in E(\mathcal{M}, \tau) \cap \mathcal{M}$ is given such that $x(\operatorname{Dom}(B)) \subseteq \operatorname{Dom}(A)$ and $A x+x B \in$ $E(\mathcal{M}, \tau) \cap \mathcal{M}$. We claim that $x(\operatorname{Dom}(A)) \subseteq \operatorname{Dom}(B)$ and $B x+x A \in E(\mathcal{M}, \tau) \cap \mathcal{M}$. Indeed, take $\xi \in \operatorname{Dom}(A)$. For $\eta \in \operatorname{Dom}(B)$ we find that

$$
\begin{aligned}
\langle x \xi, B \eta\rangle & =\langle\xi, x B \eta\rangle \\
& =\langle\xi, A x \eta+x B \eta\rangle-\langle\xi, A x \eta\rangle \\
& =\left\langle(A x+x B)^{*} \xi, \eta\right\rangle-\langle x A \xi, \eta\rangle .
\end{aligned}
$$

Since $B$ is self-adjoint, this implies that $x \xi \in \operatorname{Dom}(B)$ and

$$
B x \xi=(A x+x B)^{*} \xi-x A \xi .
$$

Moreover, this shows that $B x \xi+x A \xi=(A x+x B)^{*} \xi$ for all $\xi \in \operatorname{Dom}(A)$ and so $B x+x A$ is closable and its closure is $(A x+x B)^{*}$. We may conclude that

$$
B x+x A=(A x+x B)^{*} \in E(\mathcal{M}, \tau) \cap \mathcal{M},
$$

which proves our claim.

Defining the operator $X$ on $\mathcal{H} \oplus \mathcal{H}$ by

$$
X=\left[\begin{array}{ll}
0 & x \\
x & 0
\end{array}\right]
$$

it may easily be verified that $X \in E\left(\mathcal{M}_{2}, \tau_{2}\right) \cap \mathcal{M}_{2}$. If $(\xi, \eta) \in \operatorname{Dom}(D)$, then $X(\xi, \eta)=$ $(x \eta, x \xi)$ and from the above observations it follows that $X(\xi, \eta) \in \operatorname{Dom}(D)$. Furthermore, for $(\xi, \eta) \in \operatorname{Dom}(D)$ we find that

$$
(D X-X D)\left[\begin{array}{l}
\xi \\
\eta
\end{array}\right]=\left[\begin{array}{c}
(A x+x B) \eta \\
(-B x-x A) \xi
\end{array}\right]
$$

Therefore, $D X-X D$ is closable with closure

$$
[D, X]=D X-X D=\left[\begin{array}{cc}
0 & A x+x B \\
-B x-x A & 0
\end{array}\right]
$$


Since $A x+x B$ and $-B x-x A$ both belong to $E(\mathcal{M}, \tau) \cap \mathcal{M}$, it follows that $[D, X] \in$ $E\left(\mathcal{M}_{2}, \tau_{2}\right) \cap \mathcal{M}_{2}$. We are now in a position to apply the result of Corollary 7.5 and we may conclude that $f(X)(\operatorname{Dom}(D)) \subseteq \operatorname{Dom}(D)$ and $[D, f(X)] \in E\left(\mathcal{M}_{2}, \tau_{2}\right) \cap \mathcal{M}_{2}$, with the corresponding norm estimates. A simple computation (using, however, the fact that the function $f$ is odd) shows that

$$
f(X)=\left[\begin{array}{cc}
0 & f(x) \\
f(x) & 0
\end{array}\right]
$$

Since $f(X)$ leaves $\operatorname{Dom}(D)$ invariant, it follows that $f(x)(\operatorname{Dom}(B)) \subseteq \operatorname{Dom}(A)$ and $f(x)(\operatorname{Dom}(A)) \subseteq \operatorname{Dom}(B)$. Moreover, for all $(\xi, \eta) \in \operatorname{Dom}(D)$ we find that

$$
[D, f(X)]\left[\begin{array}{l}
\xi \\
\eta
\end{array}\right]=\left[\begin{array}{c}
(A f(x)+f(x) B) \eta \\
(-B f(x)-f(x) A) \xi
\end{array}\right] .
$$

Since $[D, f(X)]$ is a bounded operator on $\mathcal{H} \oplus \mathcal{H}$, this implies that $A f(x)+f(x) B$ and $-B f(x)-f(x) A$ are both closable, with bounded closures, and

$$
[D, f(X)]=\left[\begin{array}{cc}
0 & A f(x)+f(x) B \\
-B f(x)-f(x) A & 0
\end{array}\right] .
$$

Now it is easy to see that $A f(x)+f(x) B \in E(\mathcal{M}, \tau) \cap \mathcal{M}$. The norm estimates (7.4) follow from the corresponding estimates for $[D, f(X)]$ given in Corollary 7.5 (but, with a possibly larger constant).

Remark 7.7. If we assume in the above proposition that the function $f \in \Psi^{-1}\left(\mathfrak{C}_{0}\right)$ is even (instead of being odd), then (7.5) becomes

$$
f(X)=\left[\begin{array}{cc}
f(x) & 0 \\
0 & f(x)
\end{array}\right] .
$$

Via the same argument we may conclude that

$$
f(x)(\operatorname{Dom}(A)) \subseteq \operatorname{Dom}(A), \quad f(x)(\operatorname{Dom}(B)) \subseteq \operatorname{Dom}(B)
$$

and that $[A, f(x)],[B, f(x)] \in E(\mathcal{M}, \tau)$, with similar norm estimates.

Acknowledgements. We thank the referee for many valuable comments.

\section{References}

1. J. ARAzy, T. J. BARton AND Y. Friedman, Operator differentiable functions, Integ. Eqns Operat. Theory 13 (1990), 461-487.

2. C. Bennett And R. Sharpley, Interpolation of operators (Academic, 1988).

3. M. S. Birman and M. Z. Solomyak, Double Stieltjes operator integrals, Probl. Math. Phys. Leningrad Univ. 1 (1966), 33-67 (in Russian). 
4. M. S. Birman And M. Z. Solomyak, Double Stieltjes operator integrals, II, Probl. Math. Phys. Leningrad Univ. 2 (1967), 26-60 (in Russian).

5. M. S. Birman And M. Z. Solomyak, Double Stieltjes operator integrals, III, Probl. Math. Phys. Leningrad Univ. 6 (1973), 27-53 (in Russian).

6. M. S. BIRMAN AND M. Z. SOLOMYAK, Spectral theory and self-adjoint operators in Hilbert space (Reidel, Dordrecht, 1987).

7. M. S. Birman And M. Z. Solomyak, Operator integration, perturbations and commutators, J. Sov. Math. 63 (1993), 129-148.

8. O. BRATteli AND D. W. RoBinson, Operator algebras and quantum statistical mechanics, I (Springer, 1979).

9. H. Brezis, Analyse fonctionelle, théorie et applications (Masson, Paris, 1983).

10. V. I. Chilin AND F. A. SukocheV, Weak convergence in non-commutative symmetric spaces, J. Operat. Theory 31 (1994), 35-65.

11. E. B. Davies, One-parameter semigroups, London Mathematical Society Monographs, Volume 15 (Academic, 1980).

12. B. DE PAgter And F. A. Sukochev, Differentiation of operator functions in noncommutative $L_{p}$-spaces, J. Funct. Analysis 212 (2004), 28-75.

13. B. De Pagter, F. A. Sukochev And H. Witvliet, Double operator integrals, J. Funct. Analysis 192 (2002), 52-111.

14. J. Dixmier, Von Neumann algebras, North-Holland Mathematical Library, Volume 27 (North-Holland, Amsterdam, 1981).

15. P. G. Dodds, T. K. Dodds And B. DE Pagter, Non-commutative Banach function spaces, Math. Z. 201 (1989), 583-597.

16. P. G. Dodds, T. K. Dodds And B. De Pagter, Non-commutative Köthe duality, Trans. Am. Math. Soc. 339 (1993), 717-750.

17. N. Dunford And J. T. Schwartz, Linear operators, II, Pure and Applied Mathematics, Volume 7 (Wiley Interscience, 1963).

18. TH. FACK And H. Kosaki, Generalized $s$-numbers of $\tau$-measurable operators, Pac. $J$. Math. 123 (1986), 269-300.

19. E. Hille AND R. S. Phillips, Functional analysis and semi-groups, American Mathematical Society Colloquium Publications, Volume 31 (American Mathematical Society, Providence, RI, 1957).

20. R. V. KAdison And J. R. Ringrose, Fundamentals of the theory of operator algebras, Volume 1 (Academic, 1983).

21. R. V. KAdison And J. R. Ringrose, Fundamentals of the theory of operator algebras, Volume 2 (Academic, 1983).

22. E. Kissin And V. S. Shulman, Classes of operator-smooth functions, I, OperatorLipschitz functions, Proc. Edinb. Math. Soc. 48 (2005), 151-173.

23. E. Kissin And V. S. Shulman, Classes of operator-smooth functions, II, Operatordifferentiable functions, Integ. Eqns Operat. Theory 49 (2004), 165-210.

24. E. Kissin AND V. S. Shulman, Classes of operator-smooth functions, III, Stable functions and Fuglede ideals, Proc. Edinb. Math. Soc. 48 (2005), 175-197.

25. S. G. Krein, Ju. I. Petunin And E. M. Semenov, Interpolation of linear operators, Translations of Mathematical Monographs, Volume 54 (American Mathematical Society, Providence, RI, 1982).

26. A. McIntosh, Functions and derivations of $C^{*}$-algebras, J. Funct. Analysis 30 (1977), 264-275.

27. E. Nelson, Notes on non-commutative integration, J. Funct. Analysis 15 (1974), 103116.

28. Th. W. Palmer, Banach algebras and the general theory of *-algebras, I, Algebras and Banach algebras (Cambridge University Press, 1994). 
29. A. PAZY, Semigroups of linear operators and applications to partial differential equations, Applied Mathematical Sciences, Volume 44 (Springer, 1983).

30. V. PELlER, Hankel operators in the perturbation theory of unbounded selfadjoint operators, in Analysis and partial differential equations, Lecture Notes in Pure and Applied Mathematics, Volume 122, pp. 529-544 (Marcel Dekker, New York, 1990).

31. R. T. Powers, A remark on the domain of an unbounded derivation on a $C^{*}$-algebra, $J$. Funct. Analysis 18 (1975), 85-95.

32. S. SAKAI, Operator algebras in dynamical systems: the theory of unbounded derivations in $C^{*}$-algebras, Encyclopedia of Mathematics and its Applications, Volume 41 (Cambridge University Press, 1991).

33. S. Stratila AND L. Zsidó, Lectures on von Neumann algebras (Editura Academiei and Abacus Press, 1979).

34. M. TAKESAKI, Theory of operator algebras, I (Springer, 1979).

35. M. TAKESAKI, Theory of operator algebras, II (Springer, 2003).

36. M. TERP, $L^{p}$ spaces associated with von Neumann algebras, Research Report, Institute for Mathematical Sciences, Copenhagen University (1981).

37. W. van Ackooij, B. De Pagter and F. A. Sukochev, Domains of infinitesimal generators of automorphism flows, J. Funct. Analysis 218 (2005), 409-424. 